\title{
Photoproduction of $K^{+} K^{-}$meson pairs on the proton
}

S. Lombardo ${ }^{18, \dagger}$ M. Battaglieri, ${ }^{22, *}$ A. Celentano, ${ }^{22}$ A. D’Angelo, ${ }^{23,36}$ R. De Vita, ${ }^{22}$ A. Filippi, ${ }^{24}$ D. I. Glazier, ${ }^{43}$ S. M. Hughes, ${ }^{42}$ V. Mathieu, ${ }^{18,19,40}$ A. Rizzo, ${ }^{23,36}$ E. Santopinto, ${ }^{22}$ I. Stankovic, ${ }^{40}$ A. P. Szczepaniak, ${ }^{18,19,40}$ D. Watts, ${ }^{42}$ L. Zana, ${ }^{42}$ S. Adhikari, ${ }^{13}$ Z. Akbar, ${ }^{14}$ H. Avakian, ${ }^{40}$ J. Ball, ${ }^{7}$ N. A. Baltzell,${ }^{40,38}$ L. Barion, ${ }^{20}$ M. Bashkanov, ${ }^{42}$ V. Batourine, ${ }^{40,28}$ I. Bedlinskiy, ${ }^{26}$ A. S. Biselli,,${ }^{11,5}$ S. Boiarinov,${ }^{40}$ W. J. Briscoe, ${ }^{16}$ V. D. Burkert,${ }^{40}$ F. Cao, ${ }^{9}$ D. S. Carman ${ }^{40}$ P. Chatagnon, ${ }^{25}$ T. Chetry, ${ }^{32}$ G. Ciullo, ${ }^{20,12}$ L. Clark, ${ }^{43}$ B. A. Clary, ${ }^{9}$ P. L. Cole, ${ }^{17}{ }^{\ddagger}$ M. Contalbrigo, ${ }^{20}$ V. Crede, ${ }^{14}$ N. Dashyan, ${ }^{47}$ E. De Sanctis, ${ }^{21}$ M. Defurne, ${ }^{7}$ A. Deur, ${ }^{40}$ S. Diehl,${ }^{9}$ C. Djalali, ${ }^{38}$ M. Dugger, ${ }^{2}$ R. Dupre,${ }^{25}$ H. Egiyan, ${ }^{40}$ M. Ehrhart, ${ }^{25}$ A. El Alaoui, ${ }^{41}$ L. El Fassi, ${ }^{29}$ P. Eugenio, ${ }^{14}$ G. Fedotov, ${ }^{32}$ G. Gavalian, ${ }^{40,30}$ Y. Ghandilyan, ${ }^{47}$ G. P. Gilfoyle,${ }^{35}$ K. L. Giovanetti ${ }^{27}$ F. X. Girod, ${ }^{40,7}$ E. Golovatch, ${ }^{37}$ R. W. Gothe, ${ }^{38}$ K. A. Griffioen, ${ }^{46}$ M. Guidal, ${ }^{25}$ L. Guo, ${ }^{13,40}$ K. Hafidii ${ }^{1}$ H. Hakobyan, ${ }^{41,47}$ N. Harrison, ${ }^{40}$ M. Hattawy, ${ }^{1}$ D. Heddle, ${ }^{8,40}$ K. Hicks, ${ }^{32}$ M. Holtrop, ${ }^{30}$ Y. Ilieva, ${ }^{38,16}$ D. G. Ireland, ${ }^{43}$ B. S. Ishkhanov, ${ }^{37}$ E. L. Isupov,${ }^{37}$ D. Jenkins, ${ }^{44}$ H. S. Jo, ${ }^{28,25}$ S. Johnston, ${ }^{1}$ K. Joo, ${ }^{9}$ M. L. Kabir, ${ }^{29}$ D. Keller, ${ }^{45}$ G. Khachatryan, ${ }^{47}$ M. Khachatryan, ${ }^{33}$ M. Khandaker, ${ }^{31,8}$ A. Kim, ${ }^{9}$ W. Kim, ${ }^{28}$ A. Klein, ${ }^{33}$ F. J. Klein, ${ }^{6}$ V. Kubarovsky, ${ }^{40,34}$ L. Lanza, ${ }^{23}$ P. Lenisa, ${ }^{20}$ K. Livingston, ${ }^{43}$ I. J. D. MacGregor, ${ }^{43}$ D. Marchand, ${ }^{25}$ N. Markov, ${ }^{9}$ B. McKinnon, ${ }^{43}$ M. D. Mestayer, ${ }^{40}$ C. A. Meyer, ${ }^{5}$ Z. E. Meziani ${ }^{39}$ M. Mirazita, ${ }^{21}$ V. Mokeev, ${ }^{40,37}$ R. A. Montgomery, ${ }^{43}$ C. Munoz Camacho, ${ }^{25}$ P. Nadel-Turonski, ${ }^{40}$ S. Niccolai, ${ }^{25}$ G. Niculescu, ${ }^{27}$ M. Osipenko, ${ }^{22}$ A. I. Ostrovidov, ${ }^{14}$ M. Paolone, ${ }^{39}$ R. Paremuzyan, ${ }^{30}$ K. Park,${ }^{40,28}$ E. Pasyuk, ${ }^{40,2}$ O. Pogorelko, ${ }^{26}$ J. W. Price, ${ }^{3}$ Y. Prok,${ }^{33,45}$ D. Protopopescu, ${ }^{43}$ M. Ripani, ${ }^{22}$ D. Riser, ${ }^{9}$ B. G. Ritchie, ${ }^{2}$ G. Rosner, ${ }^{43}$ F. Sabatié, ${ }^{7}$ C. Salgado, ${ }^{31}$ R. A. Schumacher,${ }^{5}$ Y. G. Sharabian,${ }^{40}$ Iu. Skorodumina, ${ }^{38,37}$ G. D. Smith, ${ }^{42}$ D. I. Sober, ${ }^{6}$ D. Sokhan, ${ }^{43}$ N. Sparveris, ${ }^{39}$ I. I. Strakovsky, ${ }^{16}$ S. Strauch, ${ }^{38,16}$ M. Taiuti, ${ }^{22,15}$ J. A. Tan, ${ }^{28}$ M. Ungaro, ${ }^{40,9,34}$ H. Voskanyan, ${ }^{47}$ E. Voutier, ${ }^{25}$ R. Wang, ${ }^{25}$ X. Wei, ${ }^{40}$ M. H. Wood, ${ }^{4,38}$ N. Zachariou, ${ }^{42}$ J. Zhang, ${ }^{45}$ and Z. W. Zhao ${ }^{10}$

(The CLAS Collaboration)

\author{
${ }^{1}$ Argonne National Laboratory, Argonne, Illinois 60439, USA \\ ${ }^{2}$ Arizona State University, Tempe, Arizona 85287-1504, USA \\ ${ }^{3}$ California State University, Dominguez. Hills, Carson, California 90747, USA \\ ${ }^{4}$ Canisius College, Buffalo, New York 14208, USA \\ ${ }^{5}$ Carnegie Mellon University, Pittsburgh, Pennsylvania 15213, USA \\ ${ }^{6}$ Catholic University of America, Washington, DC 2006, USA \\ ${ }^{7}$ IRFU, CEA, Université Paris-Saclay, F-91191 Gif-sur-Yvette, France \\ ${ }^{8}$ Christopher Newport University, Newport News, Virginia 23606, USA \\ ${ }^{9}$ University of Connecticut, Storrs, Connecticut 06269, USA \\ ${ }^{10}$ Duke University, Durham, North Carolina 27708-0305, USA \\ ${ }^{11}$ Fairfield University, Fairfield, Connecticut 06824, USA \\ ${ }^{12}$ Università di Ferrara, 44121 Ferrara, Italy \\ ${ }^{13}$ Florida International University, Miami, Florida 33199, USA \\ ${ }^{14}$ Florida State University, Tallahassee, Florida 32306, USA \\ ${ }^{15}$ Università di Genova, 16146 Genova, Italy \\ ${ }^{16}$ The George Washington University, Washington, DC 20052, USA \\ ${ }^{17}$ Idaho State University, Pocatello, Idaho 83209, USA \\ ${ }^{18}$ Physics Department and Nuclear Theory Center, Indiana University, Bloomington, Indiana 47405, USA \\ ${ }^{19}$ Center for Exploration of Energy and Matter, Indiana University, Bloomington, Indiana 47403, USA \\ ${ }^{20}$ INFN, Sezione di Ferrara, 44100 Ferrara, Italy \\ ${ }^{21}$ INFN, Laboratori Nazionali di Frascati, 00044 Frascati, Italy \\ ${ }^{22}$ INFN, Sezione di Genova, 16146 Genova, Italy \\ ${ }^{23}$ INFN, Sezione di Roma Tor Vergata, O0133 Rome, Italy \\ ${ }^{24}$ INFN, Sezione di Torino, 10125 Torino, Italy \\ ${ }^{25}$ Institut de Physique Nucléaire, CNRS/IN2P3 and Université Paris Sud, F-91406 Orsay, France \\ ${ }^{26}$ Institute of Theoretical and Experimental Physics, Moscow 117259, Russia \\ ${ }^{27}$ James Madison University, Harrisonburg, Virginia 22807, USA \\ ${ }^{28}$ Kyungpook National University, Daegu 41566, Republic of Korea \\ ${ }^{29}$ Mississippi State University, Starkville, Mississippi 39762-5167, USA \\ ${ }^{30}$ University of New Hampshire, Durham, New Hampshire 03824-3568, USA \\ ${ }^{31}$ Norfolk State University, Norfolk, Virginia 23504, USA \\ ${ }^{32}$ Ohio University, Athens, Ohio 45701, USA
}




\author{
${ }^{33}$ Old Dominion University, Norfolk, Virginia 23529, USA \\ ${ }^{34}$ Rensselaer Polytechnic Institute, Troy, New York 12180-3590, USA \\ ${ }^{35}$ University of Richmond, Richmond, Virginia 23173, USA \\ ${ }^{36}$ Università di Roma Tor Vergata, 00133 Rome, Italy \\ ${ }^{37}$ Skobeltsyn Institute of Nuclear Physics, Lomonosov Moscow State University, 119234 Moscow, Russia \\ ${ }^{38}$ University of South Carolina, Columbia, South Carolina 29208, USA \\ ${ }^{39}$ Temple University, Philadelphia, Pennsylvania 19122, USA \\ ${ }^{40}$ Thomas Jefferson National Accelerator Facility, Newport News, Virginia 23606, USA \\ ${ }^{41}$ Universidad Técnica Federico Santa María, Casilla 110-V Valparaíso, Chile \\ ${ }^{42}$ Edinburgh University, Edinburgh EH9 3JZ, United Kingdom \\ ${ }^{43}$ University of Glasgow, Glasgow G12 8QQ, United Kingdom \\ ${ }^{44}$ Virginia Tech, Blacksburg, Virginia 24061-0435, USA \\ ${ }^{45}$ University of Virginia, Charlottesville, Virginia 22901, USA \\ ${ }^{46}$ College of William and Mary, Williamsburg, Virginia 23187-8795, USA \\ ${ }^{47}$ Yerevan Physics Institute, 375036 Yerevan, Armenia
}

(Received 7 August 2018; published 27 September 2018)

\begin{abstract}
The exclusive reaction $\gamma p \rightarrow p K^{+} K^{-}$was studied in the photon energy range 3.0-3.8 GeV and momentum transfer range $0.6<-t<1.3 \mathrm{GeV}^{2}$. Data were collected with the CLAS detector at the Thomas Jefferson National Accelerator Facility. In this kinematic range the integrated luminosity was approximately $20 \mathrm{pb}^{-1}$. The reaction was isolated by detecting the $K^{+}$and the proton in CLAS, and reconstructing the $K^{-}$via the missing-mass technique. Moments of the dikaon decay angular distributions were extracted from the experimental data. Besides the dominant contribution of the $\phi$ meson in the $P$ wave, evidence for $S-P$ interference was found. The differential production cross sections $d \sigma / d t$ for individual waves in the mass range of the $\phi$ resonance were extracted and compared to predictions of a Regge-inspired model. This is the first time the $t$-dependent cross section of the $S$-wave contribution to the elastic $K^{+} K^{-}$photoproduction has been measured.
\end{abstract}

DOI: 10.1103/PhysRevD.98.052009

\section{INTRODUCTION}

Data on light quark mesons comes mainly from hadron induced reactions, e.g., by using $K, \pi, p$ or $\bar{p}$ beams, from decays of heavy mesons and more recently from experiments making use of electromagnetic probes. Thanks to the recent advances in producing high-intensity and high-quality tagged, polarized photon beams, meson photoproduction is becoming a valuable tool to study conventional and exotic mesons. At lower energies, e.g., near single or double meson production thresholds, high quality data have been accumulated by the CB-ELSA [1-3],

\footnotetext{
*Corresponding author. marco.battaglieri@ge.infn.it

Present address: Cornell University, Ithaca, New York 14850, USA.

${ }^{\ddagger}$ Present address: Lamar University, Beaumont, Texas 77710 , USA.

${ }^{\S}$ Present address: Idaho State University, Pocatello, Idaho 83209, USA.

Published by the American Physical Society under the terms of the Creative Commons Attribution 4.0 International license. Further distribution of this work must maintain attribution to the author(s) and the published article's title, journal citation, and DOI. Funded by SCOAP.
}

CB-MAMI [4-6] and LEPS [7-9] experiments, while at higher energies, photoproduction data have come from the CLAS [10-12] experiment at Jefferson Lab. Moreover, two new programs, GLUEX [13] and MesonEx [14] have just been launched in the same laboratory. A typical meson photoproduction data set from past experiments in the energy range below $20 \mathrm{GeV}$, typical for meson spectroscopy, has tens of thousands of events, and only a few topologies have been studied [15]. For comparison, the data samples from the $g 11$ run at CLAS used here exceed the existing sets in many channels by at least an order of magnitude, and several reconstructed topologies are available for a comprehensive study [16]. Specifically, two-pseudoscalar meson photoproduction (two-pion and two-kaon) offers the possibility of investigating various aspects of the light meson resonance spectrum. Two-pion is the main decay mode of the lowest isoscalar tensor, the $f_{2}(1270)$ resonance, and it is the only known hadronic decay mode of the lowest isovector-vector resonance, the $\rho(770)$. The two-kaon channel is the main decay mode of the isoscalar-vector $\phi(1020)$ and a possible subthreshold decay of the isoscalar-scalar $f_{0}(980)$ and the isovectorscalar $a_{0}(980)$. Both the two-pion and two-kaon decay modes couple to the isoscalar-scalar channel, which contains the $f_{0}(500)$ and $f_{0}(980)$ resonances [17] and a few 
more resonances with masses above $1 \mathrm{GeV}$ that are not yet well understood; for example, the $f_{0}(500)$ meson, which is now well established [18-20], but does not fit the naive quark model classification. The $f_{0}(980)$ is similarly difficult to classify and its composition is affected by proximity to the $K \bar{K}$ threshold. These states have been the subject of extensive investigations $[21,22]$ since their observation in photon-induced reactions can provide insights into their internal structure.

In this paper we present results of the analysis of $K^{+} K^{-}$ photoproduction in the photon energy range $3.0-3.8 \mathrm{GeV}$ and momentum transfer squared $-t$ between $0.6 \mathrm{GeV}^{2}$ and $1.3 \mathrm{GeV}^{2}$, where the dikaon effective mass $M_{K^{+} K^{-}}$varies from 0.990 to $1.075 \mathrm{GeV}$. Two-kaon photoproduction data are very scarce $[23,24]$; only recently CLAS published an extensive study on $\phi$ photoproduction in the same energy range [25]. We have focused on this mass region because it is dominated by the production of the $\phi(1020)$ resonance that decays to the two kaons in the $P$ wave, and thus a partial wave analysis based on the lower $(S$ and $P$ ) waves efficiently describes it. To describe the higher mass region would require a higher number of partial waves, and this is not included in this study. Angular distributions of photoproduced mesons and related observables, such as the spherical harmonic moments and the spin density matrix elements, are the most effective tools for studying individual partial waves. For example, interference between the $S$ wave and the dominant $P$ wave was first discovered in the moment analysis of $K^{+} K^{-}$photoproduction on hydrogen in the experiments performed at DESY [26] and Daresbury [27]. More recently $\mathrm{LHCb}$ studied the dikaon system via $B_{s} \rightarrow J / \Psi K K$ decay reporting an $S$-wave fraction of $\sim 1 \%-2 \%$ for $M_{K^{+} K^{-}}$around the $\phi$ meson mass [28]. In this work we applied the same methodology used in the analysis of two-pion photoproduction to the same data set [29,30], and we refer the reader to those works for a detailed description of the analysis procedure.

This paper is organized as follows. In the next section we give a summary of the experimental setup and data analysis. Extraction of the angular moments of the twokaon system is described in Sec. III. The fit of a phenomenological model to the extracted moments is described in Sec. IV, where we also present results of the partial wave analysis, including the extracted differential cross sections for each partial wave, and a physics interpretation. A summary of the results is given in Sec. V.

\section{EXPERIMENTAL PROCEDURES AND DATA ANALYSIS}

\section{A. The photon beam and the target}

The measurement was performed with the CLAS detector [31] in Hall B at Jefferson Lab with a bremsstrahlung photon beam produced by a continuous $60 \mathrm{nA}$ electron beam of energy $E_{0}=4.02 \mathrm{GeV}$ impinging on a gold foil of thickness $8 \times 10^{-5}$ radiation lengths. A bremsstrahlung tagging system [32] with a photon energy resolution of $0.1 \%, E_{0}$ was used to tag photons in the energy range from $1.6 \mathrm{GeV}$ to a maximum energy of $3.8 \mathrm{GeV}$. In this analysis only the high-energy part of the photon spectrum, ranging from 3.0 to $3.8 \mathrm{GeV}$, was used. The $e^{+} e^{-}$pairs produced by interactions of the photon beam on an additional thin gold foil were used to continuously monitor the photon flux during the experiment. Absolute normalization was obtained by comparing the $e^{+} e^{-}$pair rate with the photon flux measured by a total absorption lead-glass counter in dedicated low-intensity runs. The energy calibration of the Hall-B tagger system was performed both by a direct measurement of the $e^{+} e^{-}$pairs produced by the incoming photons and by applying an over-constrained kinematic fit to the reaction $\gamma p \rightarrow p \pi^{+} \pi^{-}$, where all particles in the final state were detected in CLAS [33]. The quality of the calibrations was checked by looking at the mass of known particles, as well as their dependence on other kinematic variables (photon energy, detected particle momenta and angles).

The target cell, a Mylar cylinder $4 \mathrm{~cm}$ in diameter and $40-\mathrm{cm}$ long, was filled by liquid hydrogen at $20.4 \mathrm{~K}$. The luminosity was obtained as the product of the target density, target length and the incoming photon flux corrected for data-acquisition dead time. The overall systematic uncertainty on the run luminosity was estimated to be approximately $10 \%$, dominated by the uncertainty of the photon flux normalization [34].

\section{B. The CLAS detector}

Outgoing hadrons were detected in the CLAS spectrometer. Momentum information for charged particles was obtained via tracking through three regions of multiwire drift chambers [35] within a toroidal magnetic field $(\sim 1.25 \mathrm{~T})$ generated by six superconducting coils. The polarity of the field was set to bend the positive particles away from the beam line into the acceptance of the detector. Time-of-flight scintillators (TOF) were used for charged hadron identification [36]. The interaction time between the incoming photon and the target was measured by the start counter (ST) [37]. This was made of 24 strips of $2.2 \mathrm{~mm}$ thick plastic scintillator surrounding the hydrogen cell with a single-ended PMT-based readout. The average time resolution of the ST strips was $\sim 300 \mathrm{ps}$.

The CLAS momentum resolution, $\sigma_{p} / p$, ranged from 0.5 to $1.0 \%$, depending on the kinematics. The detector geometrical acceptance for each positive particle in the relevant kinematic region was about $40 \%$. It was somewhat less for low-energy negative hadrons, which could be lost at forward angles because their paths were bent toward the beam line and out of the acceptance by the toroidal field. Coincidences between the photon tagger and the CLAS detector triggered the recording of the events. The trigger in CLAS required a coincidence between the TOF and the ST in at least two sectors, in order to select reactions with at 
least two charged particles in the final state. A total integrated luminosity of $70 \mathrm{pb}^{-1}\left(\sim 20 \mathrm{pb}^{-1}\right.$ in the range $3.0<E_{\gamma}<3.8 \mathrm{GeV}$ ) was accumulated in 50 days of data taking in 2004.

\section{Data analysis and reaction identification}

The raw data were passed through the standard CLAS reconstruction software to determine the four-momenta of the detected particles. In this phase of the analysis, corrections were applied to account for the energy loss of charged particles in the target and surrounding materials, misalignments of the drift chamber positions, and uncertainties in the value of the toroidal magnetic field.

The reaction $\gamma p \rightarrow p K^{+} K^{-}$was isolated by detecting the proton and the $K^{+}$in the CLAS spectrometer, while the $K^{-}$ was reconstructed from the four-momenta of the detected particles by using the missing-mass technique. A combination of drift chambers and TOF information allowed for the identification of the kaon band in the $\beta$ vs $p$ plane for positive charged particles. More details, as well as the resulting $\mathrm{K}^{+}$missing mass spectrum for the reaction $\gamma p \rightarrow K^{+} X$ can be found in Ref. [34]. The exclusivity of the reaction was ensured by retaining events within $3 \sigma$ around the missing $K^{-}$peak $(492 \mathrm{MeV} \pm 30 \mathrm{MeV})$. This cut kept the contamination from pion misidentification and multikaon background to a minimum $(\sim 7 \%)$ for events in the dikaon mass range of interest for this analysis $\left(0.990 \mathrm{GeV}<M_{K^{+} K^{-}}<1.075 \mathrm{GeV}\right)$. Figure 1 shows the $K^{-}$missing mass. The background below the kaon peak appears as a smooth contribution to the $K^{+} K^{-}$ invariant mass that can be accounted for by fitting and subtracting a polynomial function. Since the focus of the paper is about the interference of the narrow $P$ wave (the $\phi$ meson) with the $S$ wave, the experimental background, as well as the projection of high mass hyperons populating the $p K^{+}$mass spectrum, enters in the $K^{+} K^{-}$mass as a smooth incoherent contribution that does not affect the results.

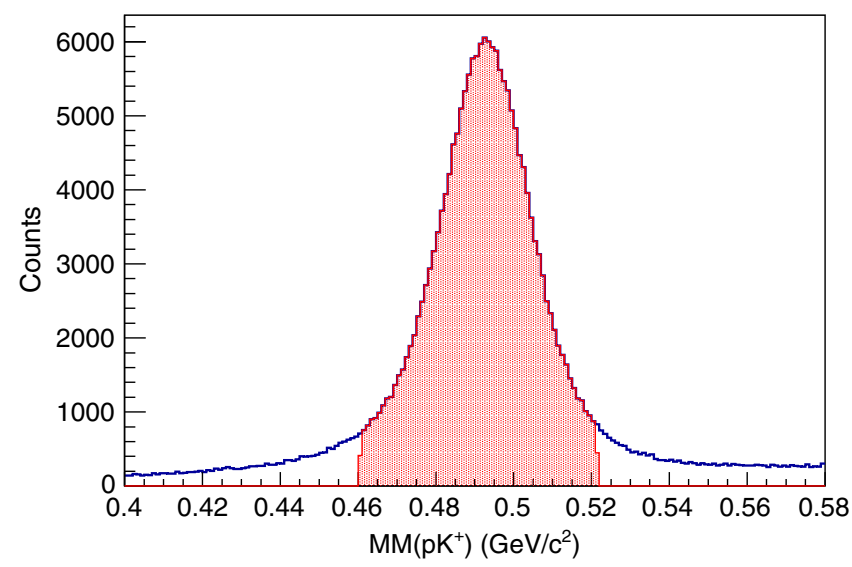

FIG. 1. Missing mass of the reconstructed $K^{-}$for the reaction $\gamma p \rightarrow p K^{+} K^{-}$. Only events in the shaded area were used in the analysis.

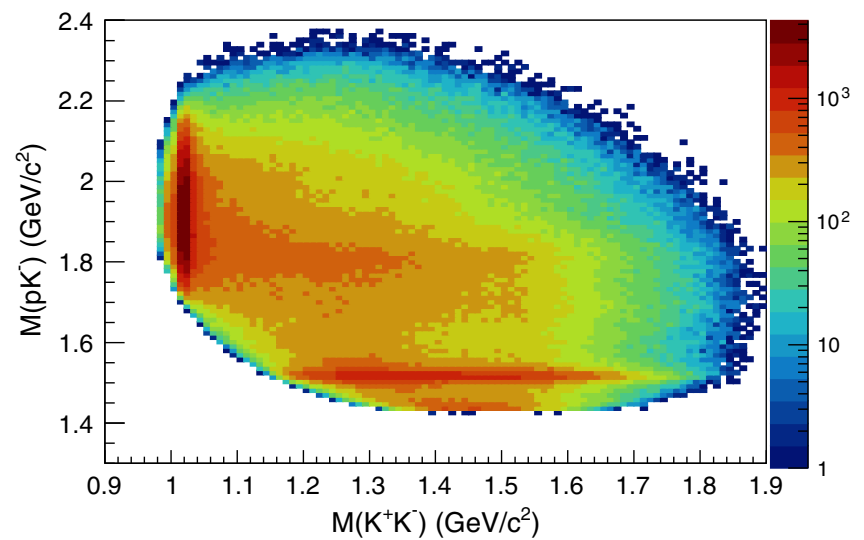

FIG. 2. Invariant mass of the $p K^{+}$system vs. invariant mass of the $K^{+} K^{-}$system. The $\phi$ meson shows up as a narrow vertical band peaked around $1 \mathrm{GeV}$, while the $\Lambda(1520)$ is visible as a horizontal band around $1.5 \mathrm{GeV}$.

To cut out edge regions in the detector acceptance, only events within a fiducial phase space volume were retained in this analysis. In the laboratory reference system, cuts were defined for the minimum hadron momentum $\left(p_{p}>0.32 \mathrm{GeV} / \mathrm{c}\right.$ and $\left.p_{K^{+}}>0.125 \mathrm{GeV} / \mathrm{c}\right)$ and the minimum angles $\left(\theta_{p}>10^{\circ}\right.$ and $\left.\theta_{K^{+}}>5^{\circ}\right)$. The fiducial cuts were defined comparing in detail the experimental data distributions with the results of the detector simulation. The minimum momentum cuts were tuned for different hadrons to take into account the energy loss as the particles pass through the target and the detector.

After all cuts, $0.2 \mathrm{M}$ events were identified as produced in the exclusive reaction $\gamma p \rightarrow p K^{+}\left(K^{-}\right)$. The other event topologies that required the $K^{-}$to be detected were not used since, in the kinematics of interest for this analysis $\left(-t<1.3 \mathrm{GeV}^{2}\right)$, the collected data were about one order of magnitude less due to the reduced detector acceptance for the inbending $K^{-}$. Figure 2 shows the invariant mass spectra of $p K^{-}$and $K^{+} K^{-}$using the reconstructed $K^{-}$ four-momentum.

The $\phi(1020)$ dominates the $K^{+} K^{-}$spectrum and the $\Lambda(1520)$ peak is visible in the mass spectrum of the $p K^{-}$ invariant mass. No overlap between the $\Lambda(1520)$ peak and the $K^{+} K^{-}$spectrum occurs for $M_{K^{+} K^{-}}<1.25 \mathrm{GeV}$. Nevertheless, a sharp cut for $M_{p K^{-}}<1.6 \mathrm{GeV}$ was applied to avoid any contamination in the meson spectrum from the $\Lambda(1520)$. A hint of excited $\Lambda$ states is visible in the bidimensional distribution but their contribution to the $K^{+} K^{-}$spectrum is very small and tends to be smooth when all hyperon states are integrated over.

\section{MOMENTS OF THE DIKAON ANGULAR DISTRIBUTIONS}

In this section we consider the analysis of spherical harmonic moments, $\left\langle Y_{L M}\right\rangle=\left\langle Y_{L M}\right\rangle\left(E_{\gamma}, t, M_{K^{+} K^{-}}\right)$, of the dikaon angular distribution defined as 


$$
\left\langle Y_{L M}\right\rangle=\sqrt{4 \pi} \int d \Omega_{K} \frac{d \sigma}{d t d M_{K^{+} K^{-}} d \Omega_{K}} Y_{L M}\left(\Omega_{K}\right),
$$

where $d \sigma$ is the four-fold differential cross section at fixed photon energy $E_{\gamma}$. Here $t$ is the momentum transfer squared between the target and the recoil proton, $M_{K^{+} K^{-}}$is the dikaon invariant mass and $Y_{L M}$ are spherical harmonics. The spherical angle $\Omega_{K}=\left(\theta_{K}, \phi_{K}\right)$ corresponds to the direction of flight of the $K^{+}$in the $K^{+} K^{-}$helicity rest frame. This is the rest frame of the $K^{+} K^{-}$pair, with the $y$ axis perpendicular to the production plane and the $z$ axis pointing in the opposite direction of the recoil nucleon momentum. In Eq. (1) the normalization has been chosen such that the $\left\langle Y_{00}\right\rangle$ moment is equal to the dikaon production differential cross section $d \sigma / d t d M_{K^{+} K^{-}}$.

There are several advantages in using moments of the angular distribution compared to a direct partial wave analysis. Moments can be expressed as bilinear in terms of the partial waves and, depending on the particular combination of $L$ and $M$, show specific sensitivity to a particular subset of them. In addition, they can be directly and unambiguously derived from the data, allowing for a quantitative comparison to the same observables calculated in specific theoretical models. Since partial wave analysis has either intrinsic mathematical ambiguities or is model dependent, it is important to extract physical observables like moments before proceeding with a model-dependent analysis [38].

The moments were extracted using two separate methods, both expanding in a model-independent set of basis functions, which were compared to the data by maximizing a likelihood function. The first of these two methods (M1) parametrized the angular distributions in terms of moments directly, while the second method (M2) used spherical harmonic partial wave amplitudes. The approximations in these two methods are dependent on the basis and on their truncation. As a check of systematics we also applied two further methods: we first binned the data and Monte Carlo simulations in all kinematical variables and divided the data by acceptance to obtain the expected angular distributions; the second used linear algebra techniques to set up an overdetermined system of equations for the moments. They provided consistent results but were not as stable or reliable as the maximum likelihood methods M1 and M2 and were not included in the final determination of the experimental moments. Detailed systematic studies using both Monte Carlo and data were performed to test the stability of the results for the different methods. A summary of these studies is reported in Appendix A. Full details regarding the procedure adopted for the moment extractions are reported in $[30,39]$.

\section{A. Detector efficiency}

The CLAS detection efficiency for the reaction $\gamma p \rightarrow$ $p K^{+} K^{-}$was obtained by means of detailed Monte Carlo simulations, which included knowledge of the full detector geometry and a realistic response to traversing particles. Events were generated according to three-particle phase space with a bremsstrahlung photon energy spectrum. A total of $96 \mathrm{M}$ events were generated in the energy range $3.0 \mathrm{GeV}<E_{\gamma}<3.8 \mathrm{GeV}$ and covered the allowed kinematic range in $-t$ and $M_{K^{+} K^{-}}$. About $19 \mathrm{M}$ events were reconstructed in the $M_{K^{+} K^{-}}$and $-t$ ranges of interest $\left(0.990 \mathrm{GeV}<M_{K}<1.365 \mathrm{GeV}, 0.6 \mathrm{GeV}^{2}<-t<1.3 \mathrm{GeV}^{2}\right)$. This corresponds to more than 400 times the statistics collected in the experiment, thereby introducing a negligible statistical uncertainty with respect to the statistical fluctuations of the data.

\section{B. Extraction of the moments via likelihood fit of experimental data}

The extraction of the moments, $\left\langle Y_{L M}\right\rangle$, was performed using the extended maximum likelihood method. As stated above, the expected theoretical yield was parametrized in terms of appropriate functions, amplitudes in one case and moments in the other. The theoretical expectation, after correction for acceptance, was compared to the experimental yield. The likelihood is then given by

$$
\mathcal{L} \sim \frac{\bar{n}_{Y_{L M}}^{n}}{n !} e^{-\bar{n}_{Y_{L M}} \Pi_{a=1}^{n}}\left[\frac{\eta\left(\tau_{a}\right) I\left(\tau_{a},\left\langle Y_{L M}\right\rangle\right)}{n\left(\left\langle\bar{Y}_{L M}\right\rangle\right)}\right] .
$$

Here $a$ represents a data event, $n$ is the number of data events in a given $\left(E_{\gamma}, t, M_{K^{+} K^{-}}\right)$bin (i.e., the fit is done independently in each bin), $\tau_{a}$ represents the set of kinematical variables of the $a$ th event (here the two-kaon decay angles), $\eta\left(\tau_{a}\right)$ is the corresponding acceptance derived by Monte Carlo simulations and $I\left(\tau_{a}\right)$ is the theoretical function representing the expected event distribution. The measure $d \tau$ includes the phase space factor and the likelihood function is normalized to the expected number of events in the bin,

$$
\bar{n}_{Y_{L M}}=\int d \tau \eta(\tau) I\left(\tau,\left\langle Y_{L M}\right\rangle\right) .
$$

This normalization integral was performed by Monte Carlo integration over the reconstructed simulated events. The parameters were extracted by minimizing a function of the form

$$
-2 \ln \mathcal{L} \propto-2 \sum_{a=1}^{n} I\left(\tau_{a},\left\langle Y_{L M}\right\rangle\right)+2 \bar{n}_{Y_{L M}} .
$$

The advantage of this approach lies in avoiding binning the data and the large uncertainties related to the corrections in regions of CLAS with vanishing efficiencies.

Comparison of the results of the two different extraction methods allows one to estimate the systematic uncertainty related to the procedure. A detailed description of the two approaches is reported in Ref. [30]. 


\section{Method comparisons and final results}

Moments derived by the different procedures agreed qualitatively. The two methods were consistent in the range of interest from $0.990 \mathrm{GeV}<M_{K^{+} K^{-}}<1.075 \mathrm{GeV}$ (and $0.6<-t<1.3 \mathrm{GeV}^{2}$ ). We do not use the region $M_{K^{+} K^{-}}>$ $1.075 \mathrm{GeV}$ to extract amplitude information because the choice of amplitude parametrization (see Sec. IVA) is only valid in proximity to the $\phi(1020)$ meson mass. The difference between the fit results of M1 and M2 was used to evaluate the systematic uncertainty associated with the moment extractions. The final results are given as the average of M1 (parametrization with moments) and M2 (parametrization with amplitudes),

$$
Y_{\text {final }}=\frac{1}{2} \sum_{i=1,2 \text { Methods }} Y_{i},
$$

where $Y$ stands for $\left\langle Y_{L M}\right\rangle\left(E_{\gamma}, t, M_{K^{+} K^{-}}\right)$. The total uncertainty $\delta Y_{\text {final }}$ in the final moments was evaluated by adding in quadrature the statistical uncertainty, $\delta Y_{\text {MINUIT }}$ as given by MINUIT, and two systematic uncertainty contributions: $\delta Y_{\text {syst fit }}$ related to the moment extraction procedure, and $\delta Y_{\text {syst norm }}$, the systematic uncertainty associated with the photon flux normalization (see Sec. II).

$$
\delta Y_{\text {final }}=\sqrt{\delta Y_{\text {MINUIT }}^{2}+\delta Y_{\text {syst fit }}^{2}+\delta Y_{\text {syst norm }}^{2}}
$$

with

$$
\begin{gathered}
\delta Y_{\text {syst fit }}=\sqrt{\sum_{i=3,4 \text { Methods }}\left(Y_{i}-Y_{\text {final }}\right)^{2}} \\
\delta Y_{\text {syst norm }}=10 \% \cdot Y_{\text {final }} .
\end{gathered}
$$

Therefore, for most of the data points, the systematic uncertainties dominate over the statistical uncertainty. Samples of the final experimental moments are shown in Figs. 3-5. The error bars include the systematic uncertainties related to the moment extraction and the photon flux normalization as discussed in Sec. III C. The whole set of moments resulting from this analysis is available in the Jefferson Lab [40] and the Durham [41] databases.

As a check of the analysis procedure, the differential cross section $d \sigma / d t$ for the $\gamma p \rightarrow p \phi(1020)$ meson was extracted by integrating the $\left\langle Y_{00}\right\rangle$ moment in each $t$ bin in the range $1.005 \mathrm{GeV}<M_{K^{+} K^{-}}<1.035 \mathrm{GeV}$ after subtracting a first-order polynomial background fitted to the data (excluding the region $1.005 \mathrm{GeV}<M_{K^{+} K^{-}}<$ $1.035 \mathrm{GeV}$ as $\left\langle\tilde{Y}_{00}\right\rangle$ is not linear due to the $\phi$ peak). The results are shown in Fig. 6. Despite the different energy binning of the various studies, the reasonable agreement within the quoted uncertainties with previous measurements $[25,27]$ gives us confidence in the accuracy of the analysis method.
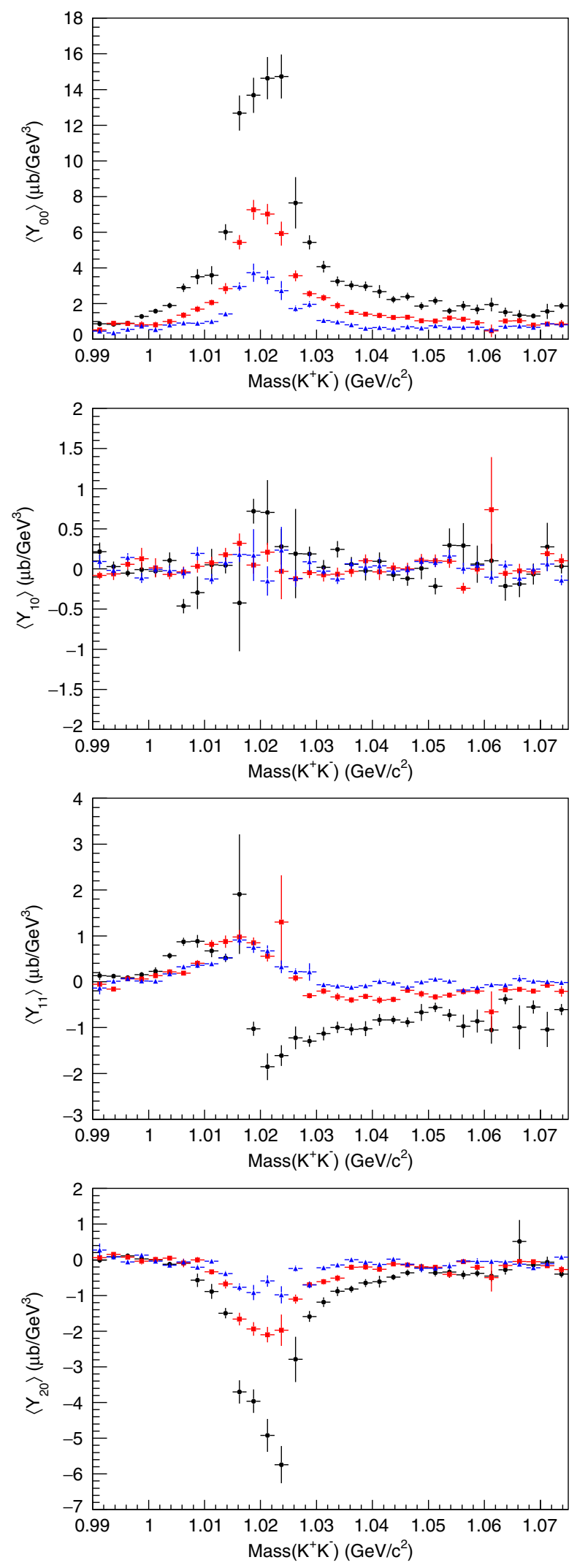

FIG. 3. Moments of the dikaon angular distributions for $3.0<E_{\gamma}<3.8 \mathrm{GeV}$ and $-t=0.45 \pm 0.05 \mathrm{GeV}^{2}$ (black), $-t=0.65 \pm$ $0.05 \mathrm{GeV}^{2}$ (red) and $-t=0.95 \pm 0.05 \mathrm{GeV}^{2}$ (blue). The error bars include both statistical and systematic uncertainties as explained in the text. 

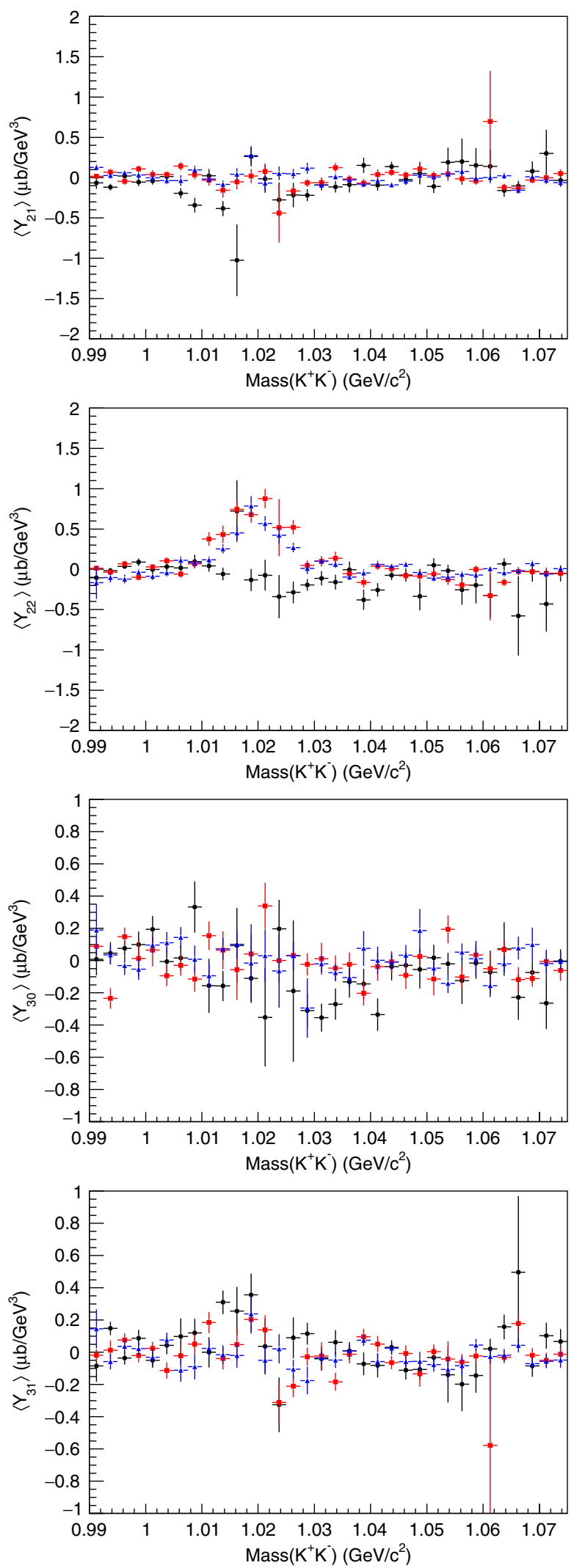

FIG. 4. Moments of the dikaon angular distributions for $3.0<E_{\gamma}<3.8 \mathrm{GeV}$ and $-t=0.45 \pm 0.05 \mathrm{GeV}^{2}$ (black), $-t=$ $0.65 \pm 0.05 \mathrm{GeV}^{2}$ (red) and $-t=0.95 \pm 0.05 \mathrm{GeV}^{2}$ (blue). The error bars include both statistical and systematic uncertainties as explained in the text.
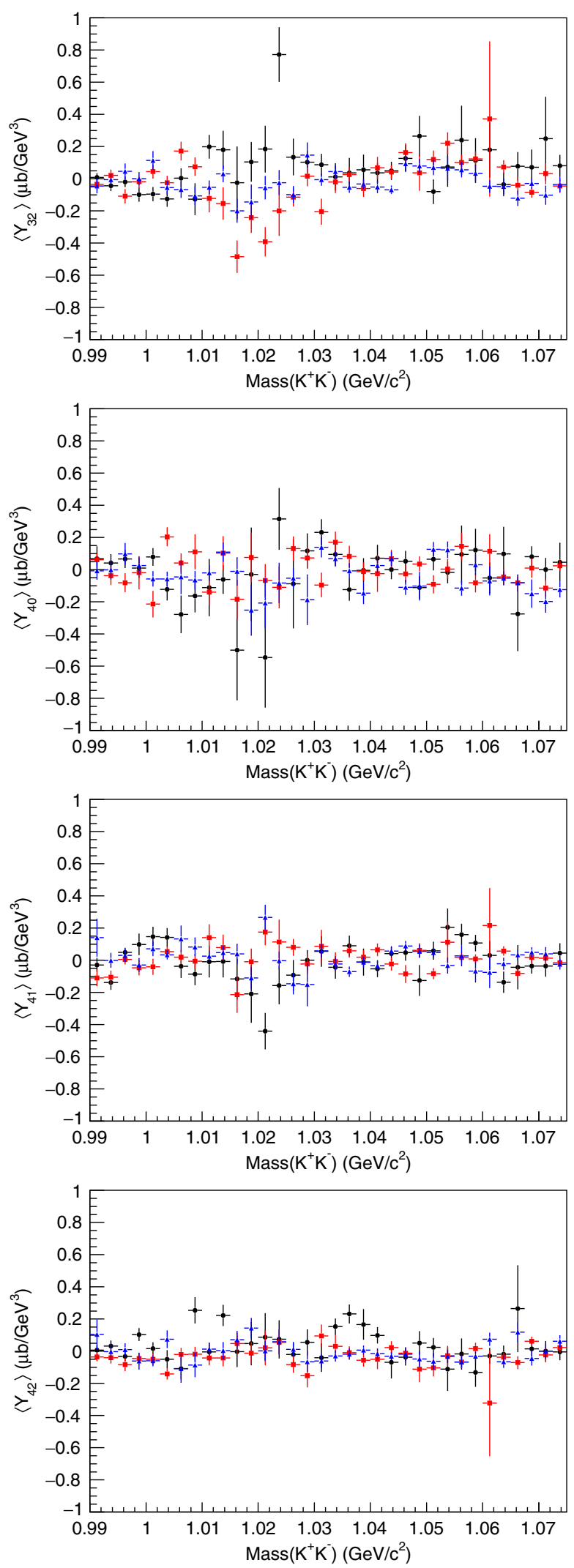

FIG. 5. Moments of the dikaon angular distributions for $3.0<E_{\gamma}<3.8 \mathrm{GeV}$ and $-t=0.45 \pm 0.05 \mathrm{GeV}^{2}$ (black), $-t=0.65 \pm 0.05 \mathrm{GeV}^{2} \quad$ (red) and $-t=0.95 \pm 0.05 \mathrm{GeV}^{2}$ (blue). The error bars include both statistical and systematic uncertainties as explained in the text. 


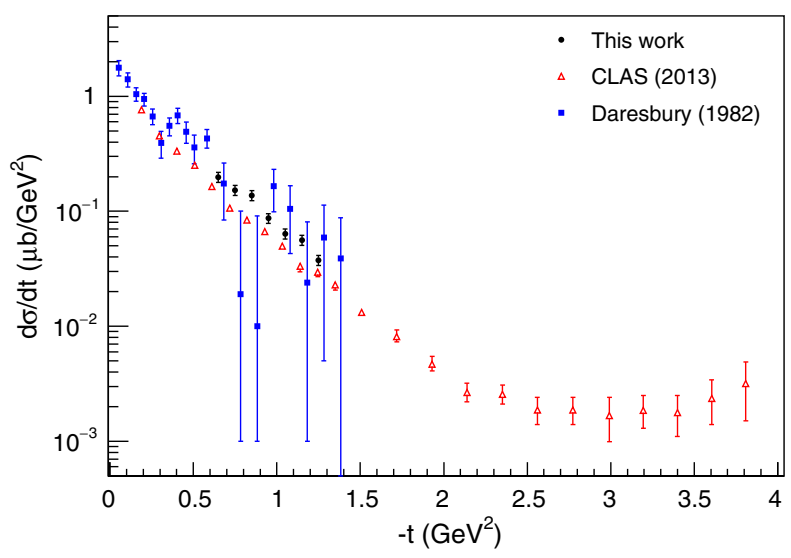

FIG. 6. Differential cross section for $3.0<E_{\gamma}<3.8 \mathrm{GeV}$ derived from the $\left\langle Y_{00}\right\rangle$ moment analysis compared with other results. The differential cross section was calculated from the $P$ wave extracted using the partial waves analysis described in Sec. IV D. The uncertainties include fit parameter uncertainties added in quadrature with a $10 \%$ systematic uncertainty from the photon flux normalization. Results of this work are compared to CLAS published results from [25] in the energy range $E_{\gamma}=3.300 \mathrm{GeV} \pm 0.015 \mathrm{GeV}$ and Daresbury data [27] in the range $2.8<E_{\gamma}<3.8 \mathrm{GeV}$.

\section{PARTIAL WAVE ANALYSIS}

In the previous section we discussed how moments of the angular distributions of the $K^{+} K^{-}$system, $\left\langle Y_{L M}\right\rangle$, were extracted from the data in each bin in photon energy, momentum transfer and dikaon mass. In this section we describe how partial waves were parametrized and extracted by fitting the experimental moments.

The production amplitudes can be written as

$f=f_{\lambda_{\gamma}, \lambda, \lambda^{\prime}}\left(s, t, M_{K^{+} K^{-}}, \Omega\right)=f_{\{\lambda\}}\left(s, t, M_{K^{+} K^{-}}, \Omega\right)$,

where $\lambda_{\gamma}, \lambda, \lambda^{\prime}$ are the helicities of the photon, target and recoil nucleons, respectively, and $M_{K^{+} K^{-}}$is the invariant mass of the $K^{+} K^{-}$system. In terms of the helicity amplitudes the cross section is given by

$$
\frac{d \sigma}{d t d M_{K^{+} K^{-}} d \Omega}\left[\frac{\mu b}{0.1 \mathrm{GeV}^{2} 2.5 \mathrm{GeV}}\right]=\Phi\left|f_{\{\lambda\}}\right|^{2}
$$

with the phase space factor $\Phi$ given by

$$
\Phi=\frac{1}{4} \frac{1.5577}{64 \pi m_{N}^{2} E_{\gamma}^{2}} \frac{\sqrt{M_{K^{+} K^{-}}^{2} / 4-m_{K}^{2}}}{2(2 \pi)^{3}}
$$
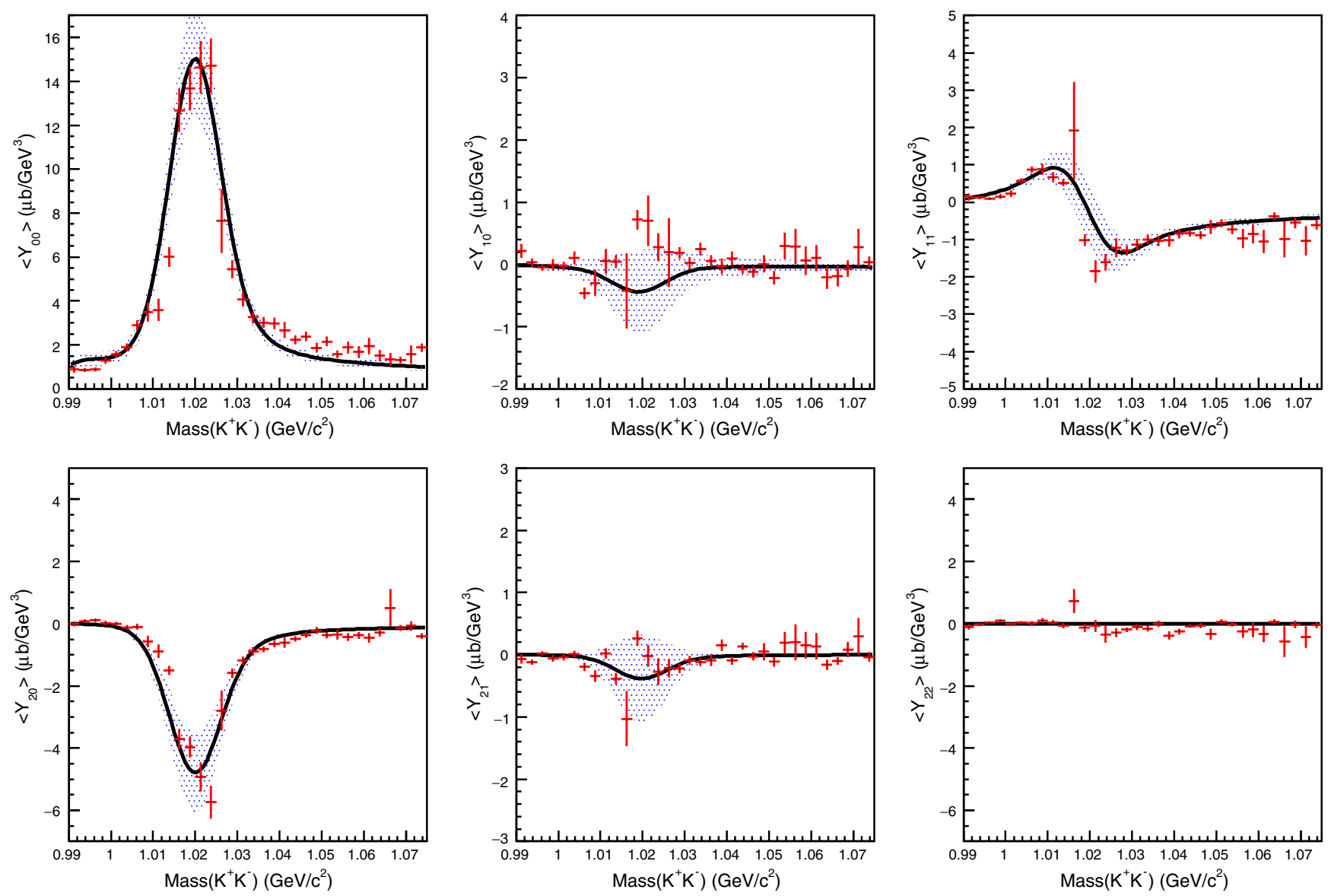

FIG. 7. Experimental moments $\left\langle Y_{L M}\right\rangle$ (red) for $0.6 \leq|t| \leq 0.7 \mathrm{GeV}^{2}$ for $L \leq 2$ and $M \leq 2$ together with the moments derived from the fitted amplitudes (black), including the $L=0$ and $L=1$ amplitudes in the fit. The shaded band indicates the associated systematic uncertainty. Under our assumptions (see text), $\left\langle Y_{22}\right\rangle=0$ in the full mass range. The solid line represents the best fit. 
where the factor of $1 / 4$ comes from averaging over the initial photon and target polarizations and all dimensional quantities enter in units of GeV. The helicity amplitudes are decomposed into partial waves $f_{\{\lambda\}}^{L M}$ in the $K \bar{K}$ channel,

$f_{\{\lambda\}}\left(s, t, M_{K^{+} K^{-}}, \Omega\right)=\sum_{L M} f_{\{\lambda\}}^{L M}\left(s, t, M_{K^{+} K^{-}}\right) Y_{L M}(\Omega)$,

so that the moments, defined in (1), are given by

$$
\frac{\left\langle Y_{L M}\right\rangle}{\Phi}=\sum_{L_{1}, M_{1}, L_{2}, M_{2} ;\{\lambda\}} c_{L_{1}, M_{1}, L_{2}, M_{2} ; L M}\left[f_{\{\lambda\}}^{L_{1} M_{1} *} f_{\{\lambda\}}^{L_{2} M_{2}}\right],
$$

with the $c$ 's proportional to a product of Clebsch-Gordan coefficients. Note that we are using the spherical basis for the spin projection $M$ and not the so-called reflectivity basis. Equation (13) is a bilinear relation between the moments derived from the data and the partial wave amplitudes. The fit minimized the difference of the right and the left sides of Eq. (13) with respect to free parameters in the amplitude parametrization. In this way, a set of moments was used to determine the amplitudes.

\section{A. Parametrization of the partial waves}

For a given $L$ and $M$, there are eight independent amplitudes, $f_{\lambda_{y}, \lambda, \lambda^{\prime}}^{L M}\left(M_{K^{+} K^{-}}\right)$, in each energy and momentum transfer bin corresponding to each combination of photon and initial and final nucleon helicity. We have only one energy bin in this analysis, so the fitted amplitudes do not depend on $E_{\gamma}$. Since the $L \geq 2$ amplitudes ( $D$ and $F$ waves) are expected to be small in the $K^{+} K^{-}$invariant mass range, we only include $S$ and $P$ partial waves. The reaction $\gamma p \rightarrow$ $p K^{+} K^{-}$was then characterized by 32 amplitudes. There were 8 amplitudes required to describe the $S$ wave depending on the two-spin projections of the photon $\left(\lambda_{\gamma}= \pm 1\right)$, the target proton $(\lambda= \pm 1 / 2)$, and the recoil proton $\left(\lambda^{\prime}= \pm 1 / 2\right)$. In addition, there were $24 P$-wave amplitudes depending also on three-spin projections of the $\phi$. However, the photon helicity was restricted to $\lambda_{\gamma}=+1$ since the other amplitudes are related by parity conservation, resulting in 16 unconstrained amplitudes. In addition, some approximations in the parametrization of the partial waves were adopted to reduce the number of free parameters in the fit as discussed below.
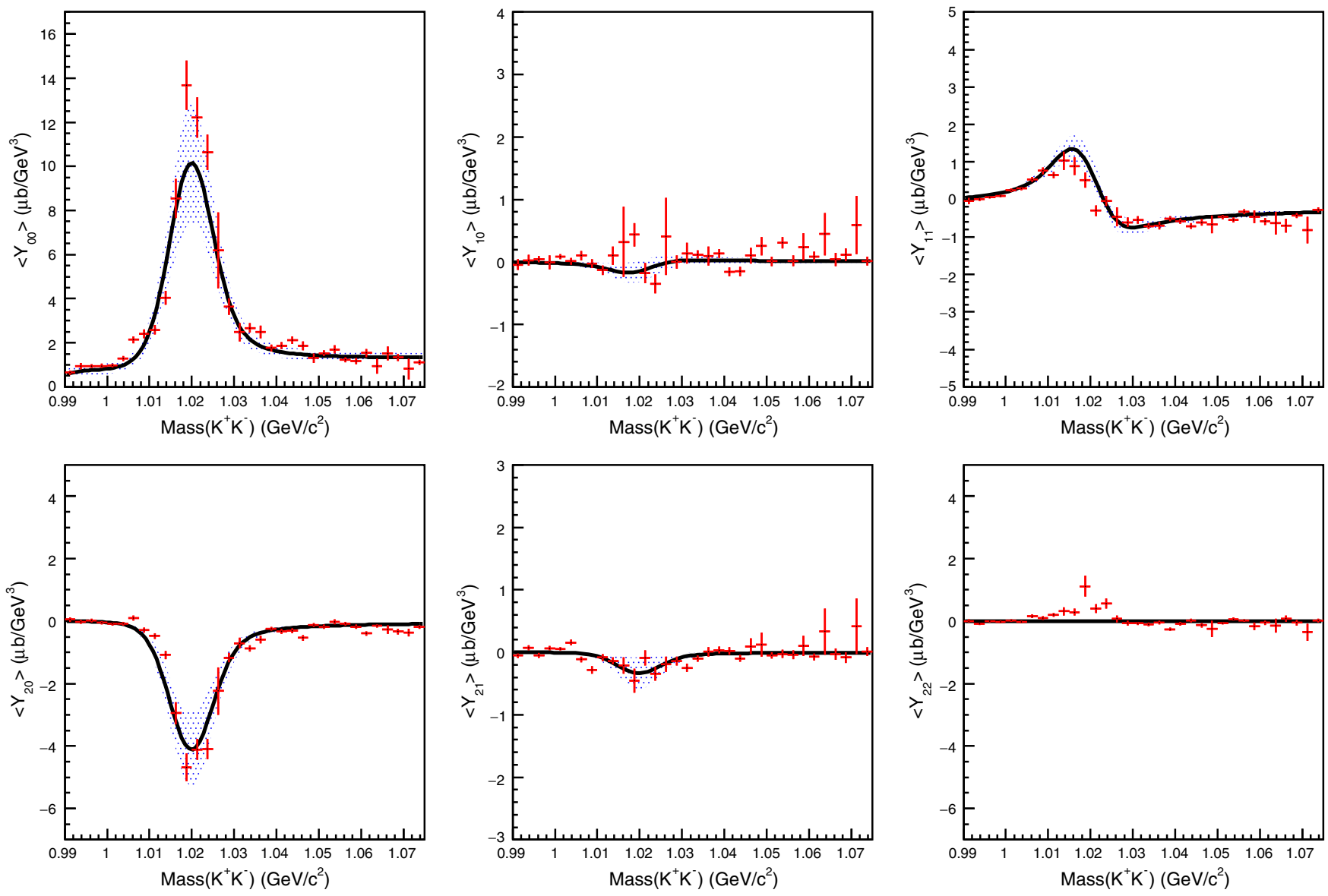

FIG. 8. Experimental moments $\left\langle Y_{L M}\right\rangle$ (red) for $0.7 \leq|t| \leq 0.8 \mathrm{GeV}^{2}$ for $L \leq 2$ and $M \leq 2$ together with the moments derived from the fitted amplitudes (black), including the $L=0$ and $L=1$ amplitudes in the fit. The shaded band indicates the associated systematic uncertainty. Under our assumptions (see text), $\left\langle Y_{22}\right\rangle=0$ in the full mass range. The solid line represents the best fit. 
In general, it is expected that the dominant amplitudes require minimal photon helicity flip, i.e.

$$
\left|f^{L 1}\right|>\left|f^{L 0}\right|,
$$

corresponding to photon helicity flip by zero and one, respectively. In the $s$-channel helicity frame, we assume the $P$-wave production $(L=1)$ is dominated by helicity nonflip amplitudes, i.e. the nonvanishing independent amplitudes are

$$
P_{+} \equiv f_{+,+,+}^{1,1}, \quad P_{-} \equiv f_{+,-,-}^{1,1},
$$

where \pm refer to helicities of the photon and the protons, e.g., ,,+++ corresponds to $\lambda_{\gamma}=+1, \lambda=+1 / 2$ and $\lambda^{\prime}=+1 / 2$. We introduced two additional amplitudes per each orbital angular momentum, to describe unit photon helicity flip,

$$
P_{0+} \equiv f_{+,+,+}^{1,0}, \quad P_{0-} \equiv f_{+,-,-}^{1,0}
$$

and

$$
S_{+} \equiv f_{+,+,+}^{0,0}, \quad S_{-} \equiv f_{+,-,-}^{0,0} .
$$

In the approximations described above, the dependence of moments on the $S$ and $P$ amplitudes is given by

$$
\begin{aligned}
& \left\langle Y_{00}\right\rangle=2\left[\left|S_{+}\right|^{2}+\left|S_{-}\right|^{2}+\left|P_{+}\right|^{2}+\left|P_{-}\right|^{2}+\left|P_{0+}\right|^{2}+\left|P_{0-}\right|^{2}\right] \\
& \left\langle Y_{10}\right\rangle=2\left[S_{+}^{*} P_{0+}+S_{-}^{*} P_{0-}+P_{0+}^{*} S_{+}+P_{0-}^{*} S_{-}\right] \\
& \left\langle Y_{11}\right\rangle=P_{+}^{*} S_{+}+P_{-}^{*} S_{-}+S_{+}^{*} P_{+}+S_{-}^{*} P_{-} \\
& \left\langle Y_{20}\right\rangle=\frac{2}{\sqrt{5}}\left[2\left|P_{0+}\right|^{2}+2\left|P_{0-}\right|^{2}-\left|P_{+}\right|^{2}-\left|P_{-}\right|^{2}\right] \\
& \left\langle Y_{21}\right\rangle=\sqrt{\frac{3}{5}}\left[P_{0+}^{*} P_{+}+P_{0-}^{*} P_{-}+P_{+}^{*} P_{0+}+P_{-}^{*} P_{0-}\right]
\end{aligned}
$$

with $\left\langle Y_{22}\right\rangle$ vanishing under our assumptions. Here we see the $\left\langle Y_{10}\right\rangle$ and $\left\langle Y_{11}\right\rangle$ moments contain information about the presence of the $S$-wave interference with the dominant $P$ wave. Thus, a nonzero $\left\langle Y_{10}\right\rangle$ or $\left\langle Y_{11}\right\rangle$ moment is an indication of a nonvanishing $S$-wave amplitude. In order for
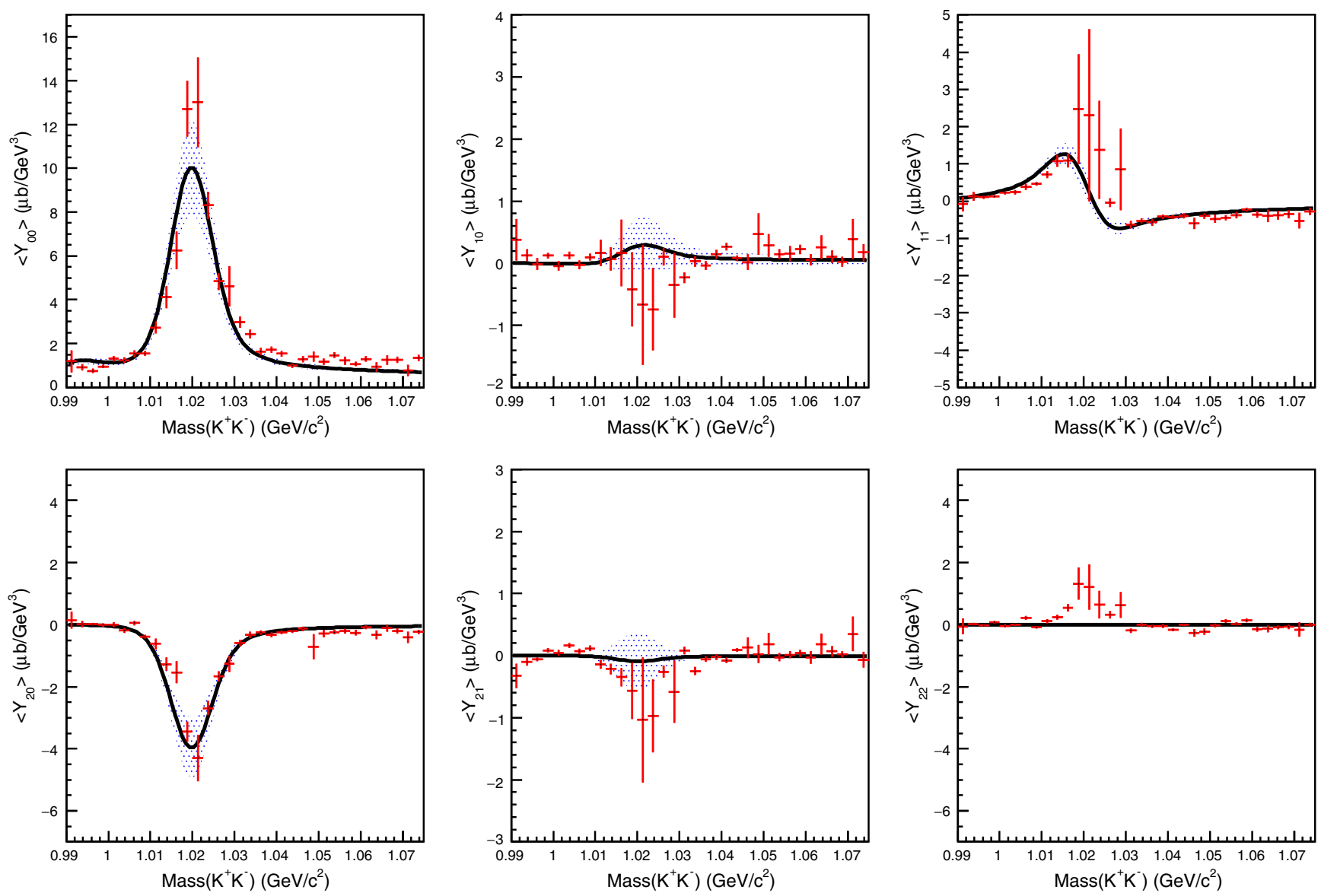

FIG. 9. Experimental moments $\left\langle Y_{L M}\right\rangle$ (red) for $0.8 \leq|t| \leq 0.9 \mathrm{GeV}^{2}$ for $L \leq 2$ and $M \leq 2$ together with the moments derived from the fitted amplitudes (black), including the $L=0$ and $L=1$ amplitudes in the fit. The shaded band indicates the associated systematic uncertainty. Under our assumptions (see text), $\left\langle Y_{22}\right\rangle=0$ in the full mass range. The solid line represents the best fit. 
the $\left\langle Y_{22}\right\rangle$ moment to be nonzero, there must be two-unit photon helicity flip amplitudes. Given that there is no significant structure in any $\left\langle Y_{22}\right\rangle$ moments of this analysis, it is justified to neglect two-unit photon helicity flip amplitudes. So far we have introduced only the nucleon helicity nonflip amplitudes. Indeed,$P$-wave nucleon helicity flip amplitudes are expected to be small (cf. Appendix B and Ref. [42]).

Without polarization information, it is difficult to separate out amplitudes differing only by the helicity of the nucleon. We did attempt to fit the data using various configurations of nucleon helicity amplitudes and found, in particular, that the $S-P$ interference signal in the $\left\langle Y_{11}\right\rangle$ moment cannot be described solely by interference between nucleon flip amplitudes. We comment on this further in Sec. IV C. We find, however, that the moments can be well described by interference between the dominant, nucleon helicity nonflip $P$ - and $S$-wave amplitudes. Details of the amplitude parametrization are given in Appendix B.

\section{B. Fit of the moments}

To account for detector resolution, the moments calculated from the amplitudes were smeared by a Gaussian function. The $\phi$ width apparent in the $\left\langle Y_{00}\right\rangle$ moment determined the smearing needed in order for the $P$-wave parametrization (with fixed $\phi$ width) to match the data. This lead to a width in the Gaussian smearing of $4 \mathrm{MeV}$, which is compatible with the CLAS detector resolution measured in other reactions [34]. We fit the moments $\left\langle Y_{L M}\right\rangle$ with $L \leq 2$ and $M \leq 2$ using up to $L=1(P)$ waves as described above. In Figs. 7-13, we present the fit results of this analysis from $0.6<-t<1.3 \mathrm{GeV}^{2}$. To properly take into account the uncertainty contributions (statistical and systematic) to the experimental moments described in Sec. III C, the two sets of moments from methods M1 and M2 were individually fit, and the fit results were averaged, obtaining the central value shown by the black line in the figures. The error band, shown as a grey area, was calculated following the same procedure adopted for the experimental moments (Sec. III C). The two lowest momentum transfer bins $0.4 \leq$ $t \leq 0.6 \mathrm{GeV}^{2}$ were excluded from the analysis because the
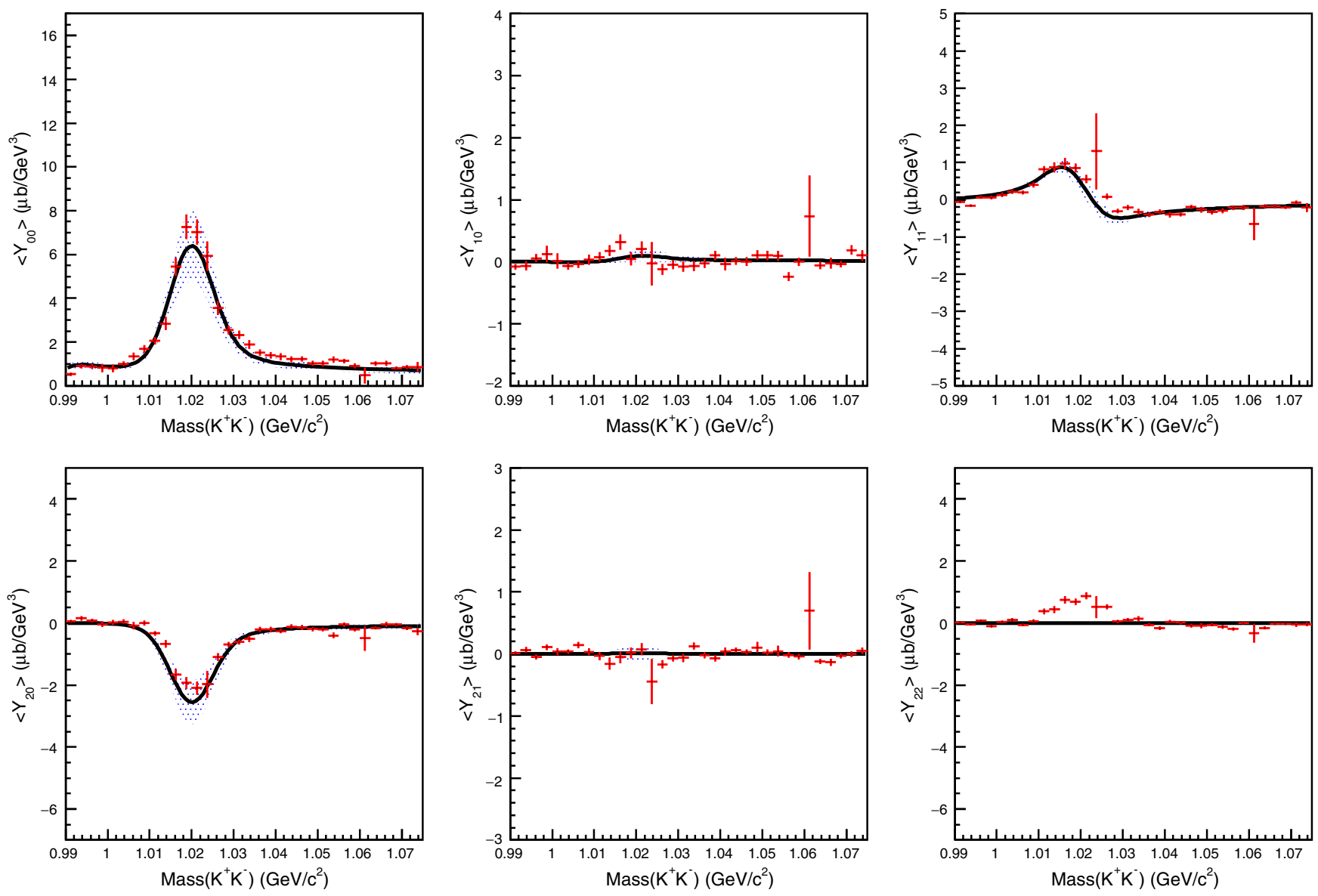

FIG. 10. Experimental moments $\left\langle Y_{L M}\right\rangle$ (red) for $0.9 \leq|t| \leq 1.0 \mathrm{GeV}^{2}$ for $L \leq 2$ and $M \leq 2$ together with the moments derived from the fitted amplitudes (black), including the $L=0$ and $L=1$ amplitudes in the fit. The shaded band indicates the associated systematic uncertainty. Under our assumptions (see text), $\left\langle Y_{22}\right\rangle=0$ in the full mass range. The solid line represents the best fit. 
moment reconstruction procedure was found not to be reliable in this region. In addition, the $\left\langle Y_{10}\right\rangle$ moment was not used to extract the $S$-wave magnitude because the procedure could not always reproduce an accurate $\left\langle Y_{10}\right\rangle$ moment based on tests performed on pseudodata.

\section{Partial wave amplitudes}

As an example, the square of the magnitude of the $S$ and $P$ partial waves derived by fit for the momentum transfer bin $0.7<-t<0.8 \mathrm{GeV}^{2}$ are shown in Fig. 14. The $S$-wave threshold enhancement provides a hint of the scalar $f_{0}(980)$ or $a_{0}(980)$ states, which have been parametrized by the exchange of the $\omega$ and $\rho$ vector mesons in the $t$ channel. The top and the middle plots show the partial waves summed over all helicities. The two bottom plots show the amplitudes for two possible values of $M=1,0$, the helicity of the dikaon system. Note that we use the wave with photon helicity $\lambda_{\gamma}=+1$ as a reference. Thus, $M=1$ corresponds to the nohelicity flip ( $s$ channel helicity conserving) amplitude, which, as expected, is the dominant one, and $M=0$ corresponds to unit photon helicity flip. The nonvanishing $\left\langle Y_{22}\right\rangle$ moments show the presence of a small two-unit helicity flip amplitude. By neglecting the $M=-1$ amplitudes, we have focused on describing the dominant structure in the $\left\langle Y_{11}\right\rangle$ and $\left\langle Y_{20}\right\rangle$ moments and reducing the number of fit parameters.

To check sensitivity to various helicity components we performed the fit in three configurations. In the first configuration we included $S$ - and $P$-wave amplitudes with vanishing photon helicity flip and unit photon helicity flip. Nucleon helicity flip amplitudes were excluded. In the second configuration, we used Regge factorization to reduce the number of independent amplitudes. Specifically, the parity relation applied to the nucleon vertex [43] reduces the number of unconstrained amplitudes by a factor of two, since $S_{+}$is related to $S_{-}, P_{+}$to $P_{-}$, and $P_{0+}$ to $P_{0-}$. Finally, in the third configuration we used the above Regge-constrained $P$-wave amplitudes and added to them the nucleon helicity flip amplitudes. In this configuration we tested if the interference signal in the moments could be described by interfering nucleon flip
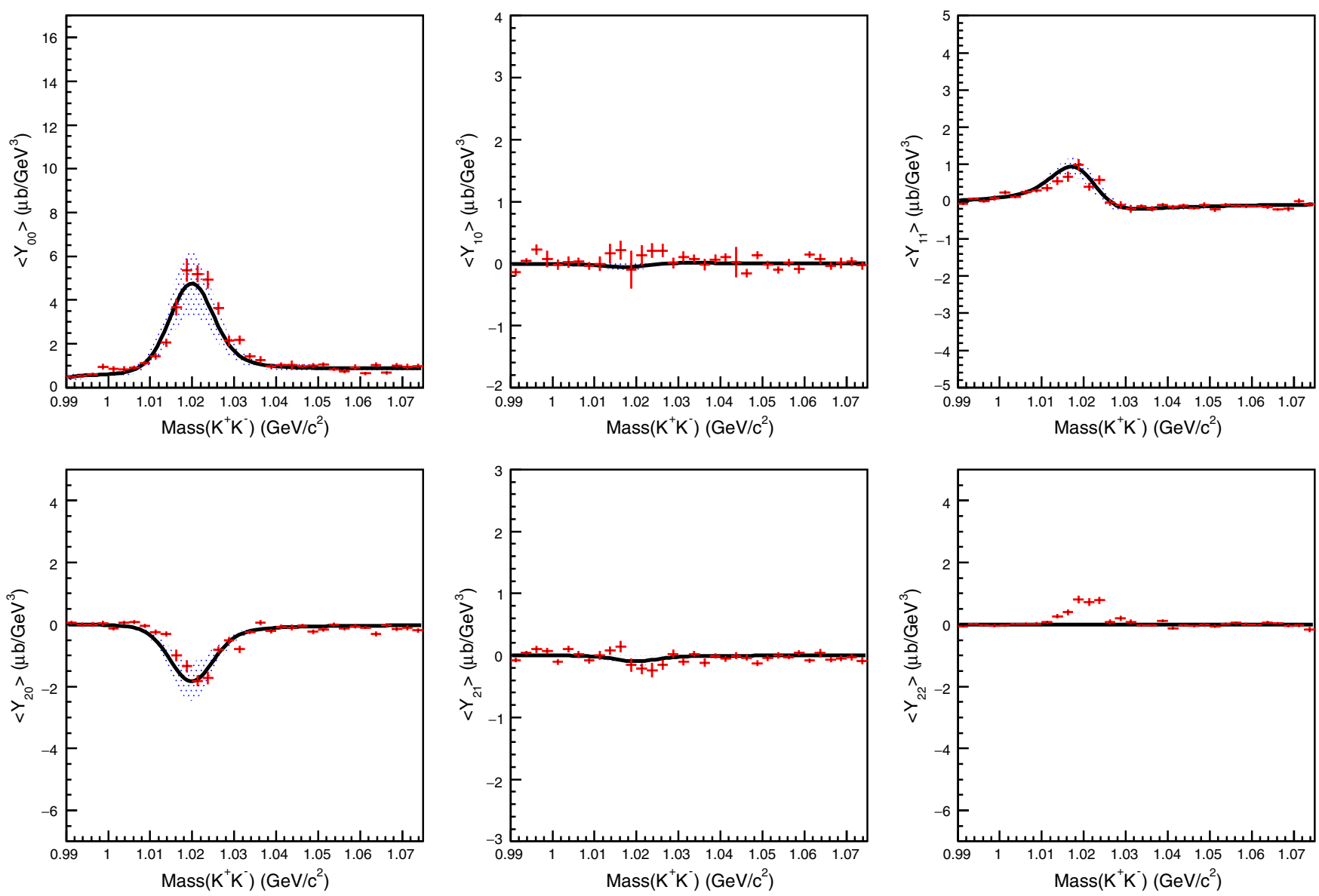

FIG. 11. Experimental moments $\left\langle Y_{L M}\right\rangle$ (red) for $1.0 \leq|t| \leq 1.1 \mathrm{GeV}^{2}$ for $L \leq 2$ and $M \leq 2$ together with the moments derived from the fitted amplitudes (black), including the $L=0$ and $L=1$ amplitudes in the fit. The shaded band indicates the associated systematic uncertainty. Under our assumptions (see text), $\left\langle Y_{22}\right\rangle=0$ in the full mass range. The solid line represents the best fit. 

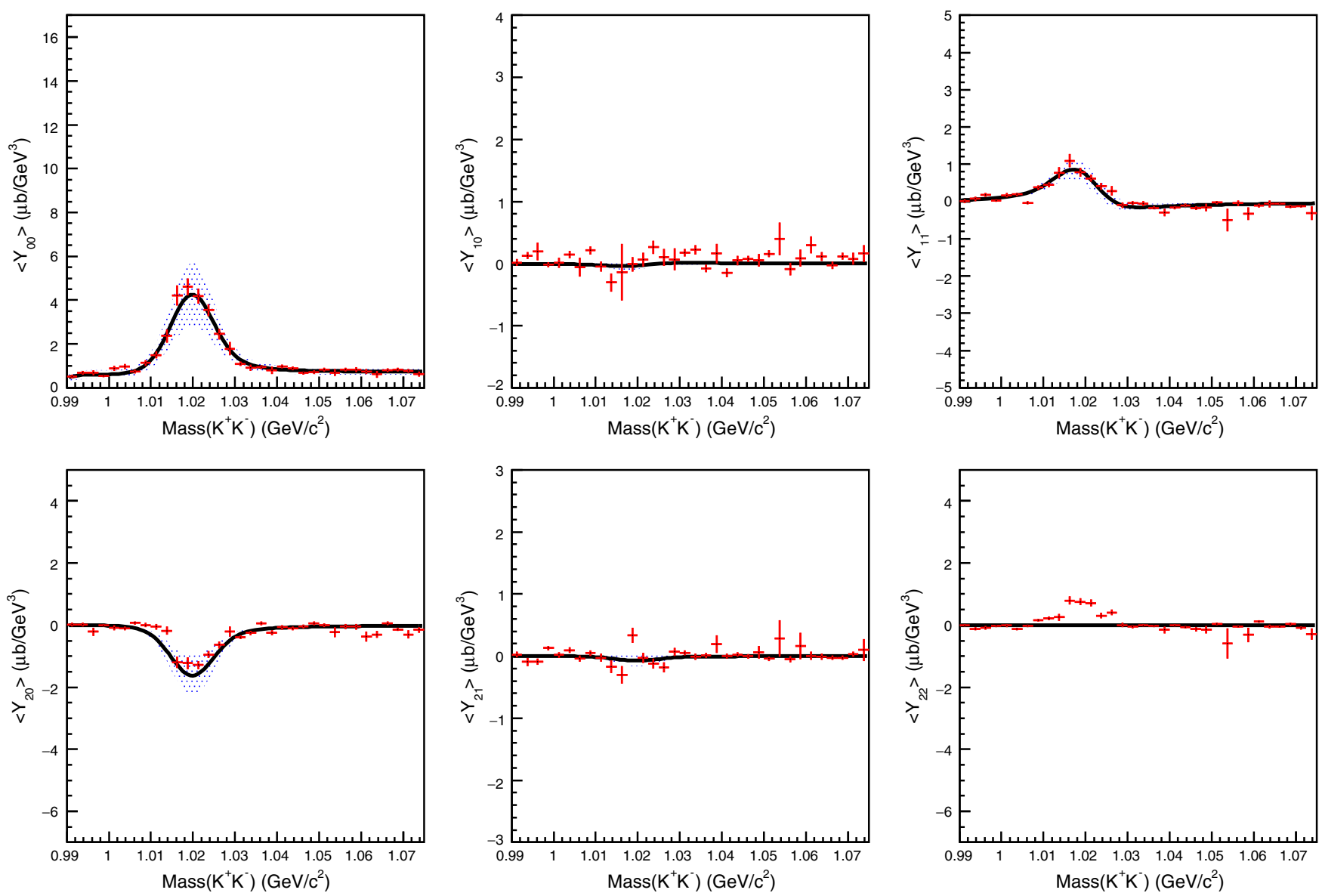

FIG. 12. Experimental moments $\left\langle Y_{L M}\right\rangle$ (red) for $1.1 \leq|t| \leq 1.2 \mathrm{GeV}^{2}$ for $L \leq 2$ and $M \leq 2$ together with the moments derived from the fitted amplitudes (black), including the $L=0$ and $L=1$ amplitudes in the fit. The shaded band indicates the associated systematic uncertainty. Under our assumptions (see text), $\left\langle Y_{22}\right\rangle=0$ in the full mass range. The solid line represents the best fit.

amplitudes by attempting to extract the nucleon helicity flip amplitudes from the $\left\langle Y_{10}\right\rangle$ and $\left\langle Y_{11}\right\rangle$ moments. Specifically, we added two nucleon helicity flip $P$-wave amplitudes $f_{++-}^{1,1}, f_{++-}^{1,0}$ and one nucleon flip $S$-wave amplitude $f_{++-}^{0,0}$. It is only necessary to consider one-half of all the nucleon flip amplitudes because the others are not independent after using the Regge factorization condition. We found that the first two configurations gave similar results, and specifically, in Figs. 7-13, we show the results obtained with the second configuration described above. In the third configuration a fit was first performed using the $\left\langle Y_{00}\right\rangle$ and $\left\langle Y_{20}\right\rangle$ moments to extract the dominant nucleon nonflip $P$ wave, while setting the nucleon flip amplitudes to zero. After fixing the strength of the nonflip $P$ wave in this way, we introduced nucleon flip $P$ and $S$ waves and added the $\left\langle Y_{10}\right\rangle$ and $\left\langle Y_{11}\right\rangle$ moments to the fit. As shown in Fig. 15, we found that the nucleon flip amplitudes cannot be large enough to significantly affect the $\left\langle Y_{11}\right\rangle$ moment. We thus conclude that the nonflip amplitudes dominate the measured moments.

\section{Differential cross sections}

Differential cross section $(d \sigma / d t)_{L}$ for individual waves can be obtained by integrating the corresponding amplitude obtained from fits to the moments. The results are shown in Figs. 16 and 17. All cross sections are found by integrating the mass region $1.0195 \pm 0.0225 \mathrm{GeV}$. It is worth noting that the magnitudes of the $S$ and $P_{0}$ waves found in this analysis (see Table I) are consistent with predictions (summarized in Table II) of a model constrained on a somewhat higher photon energy data $[24,26,27]$. The discrepancy can be explained by the different $-t$ integration range.

\section{E. Uncertainty evaluation}

The final uncertainty was computed as the sum in quadrature of the statistical uncertainty of the fit, and 

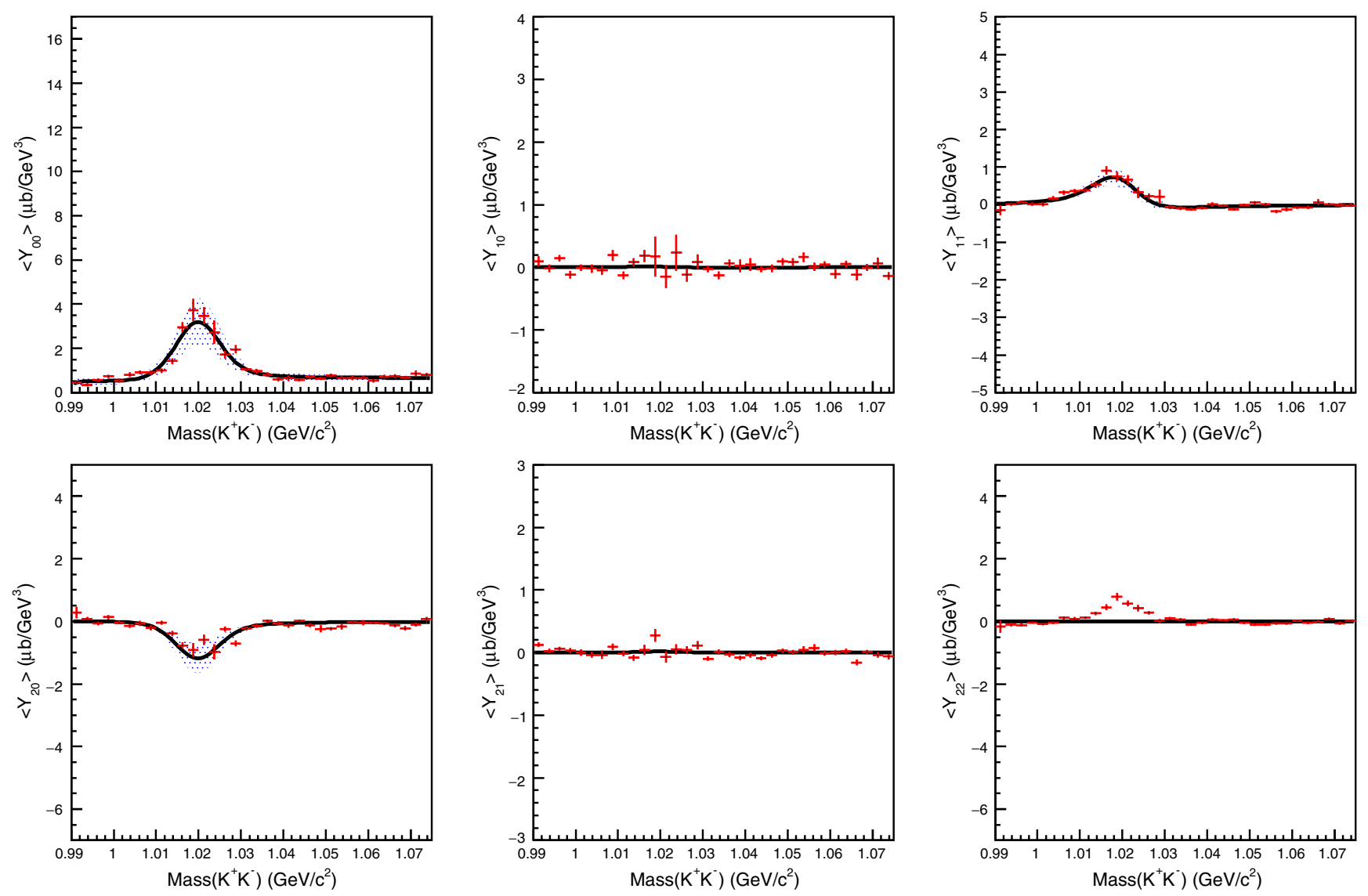

FIG. 13. Experimental moments $\left\langle Y_{L M}\right\rangle$ (red) for $1.2 \leq|t| \leq 1.3 \mathrm{GeV}^{2}$ for $L \leq 2$ and $M \leq 2$ together with the moments derived from the fitted amplitudes (black), including the $L=0$ and $L=1$ amplitudes in the fit. The shaded band indicates the associated systematic uncertainty. Under our assumptions (see text), $\left\langle Y_{22}\right\rangle=0$ in the full mass range. The solid line represents the best fit.

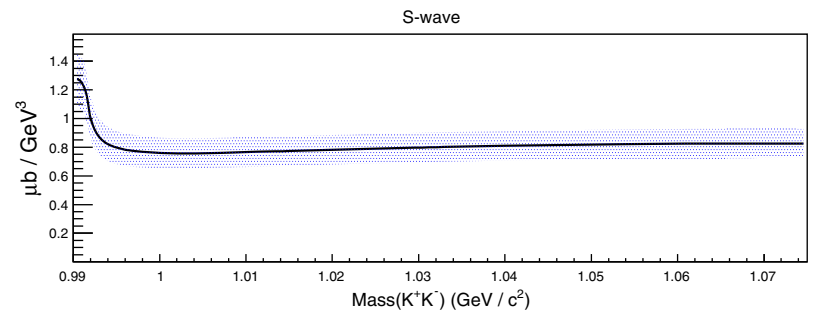

P-wave
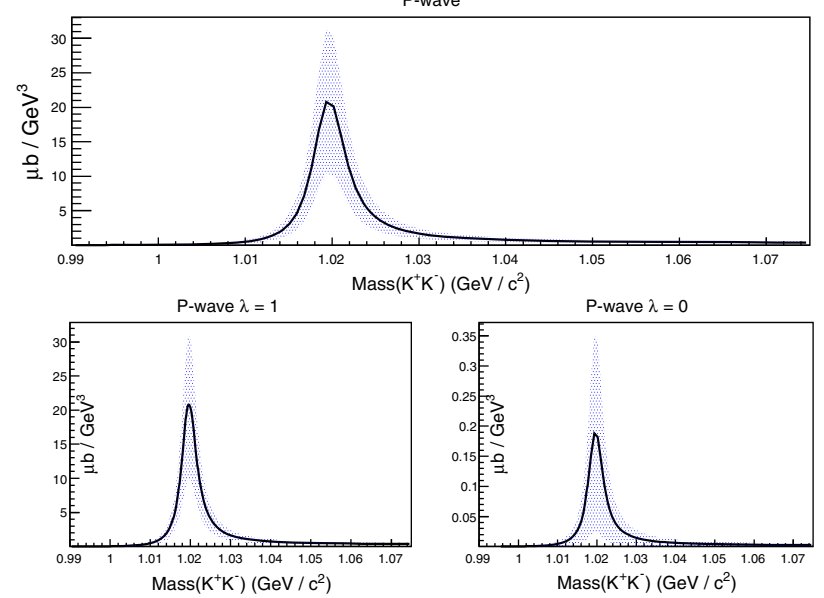

FIG. 14. Magnitudes of the $S$ and $P$ partial waves along with twospin projections of the $P$ wave $\left(\lambda_{K K}=1,0\right)$ in the $0.7 \leq t \leq$ $0.8 \mathrm{GeV}^{2}$ bin determined by fitting to the experimental moments. two systematic uncertainty contributions: the first related to the moment extraction procedure, evaluated as the variance of the two fit results, and the second associated with the photon flux normalization estimated

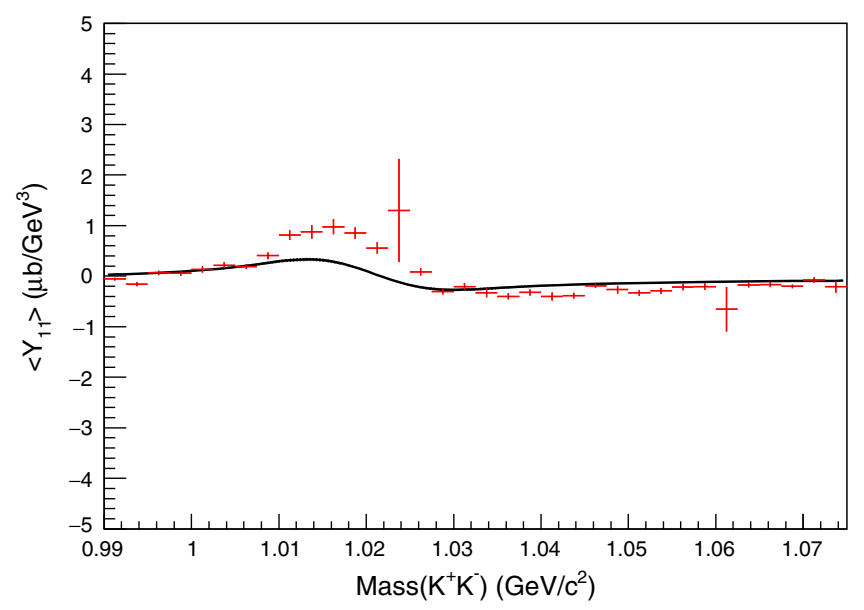

FIG. 15. Fit of $\left\langle Y_{11}\right\rangle$ moment with the nucleon flip amplitude alone. The bad agreement indicates the nonflip amplitudes dominate the measured moments. 


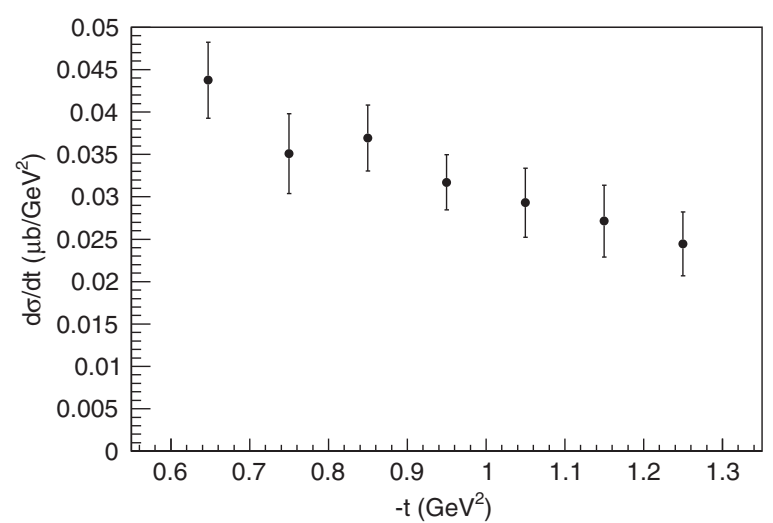

FIG. 16. Differential cross section obtained from integrating the $S$-wave magnitude in the $M_{K^{+} K^{-}}$range $1.0195 \pm$ $0.0225 \mathrm{GeV}$.

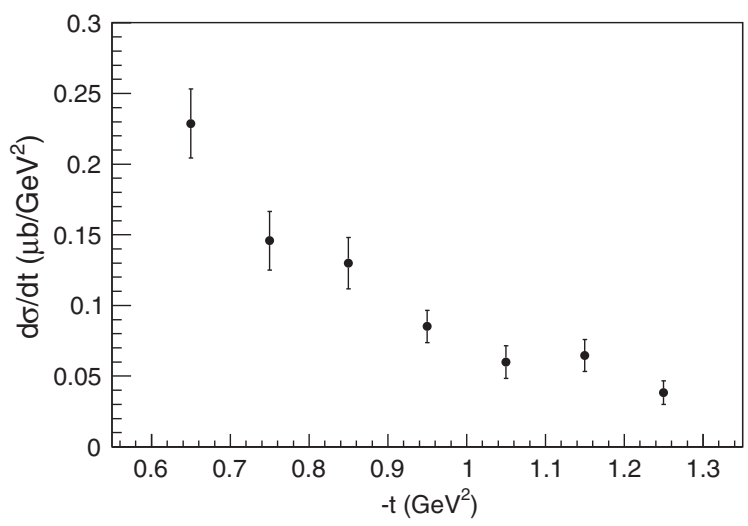

FIG. 17. Differential cross section obtained from integrating the $P$-wave magnitude in the $M_{K^{+} K^{-}}$range $1.0195 \pm$ $0.0225 \mathrm{GeV}$.

to be $10 \%$. The central values and uncertainties for all of the observables of interest discussed in the next sections were derived from the fit results with the same procedure.

TABLE I. Cross sections in nb obtained from this analysis by integrating the $S$ - and $P$-wave magnitudes in the $M_{K^{+} K^{-}}$range $1.0195 \pm 0.0225 \mathrm{GeV}$ in the single momentum transfer bin $0.6 \leq-t \leq 0.7 \mathrm{GeV}^{2}$.

\begin{tabular}{lc}
\hline \hline Photon energy & $3.0-3.8 \mathrm{GeV}$ \\
\hline Total cross section & 27.2 \\
Sum of $P$ waves & $22.9 \pm 2.4$ \\
$P_{0}$ wave & $1.9 \pm 0.6$ \\
$S$ wave & $4.3 \pm 0.45$ \\
\hline \hline
\end{tabular}

TABLE II. Cross sections in nb obtained from integrating the $S$ and $P$ waves from the Regge model of [44]. The results shown are integrated over $-t$ up to $1.5 \mathrm{GeV}^{2}$ and the $M_{K \bar{K}}$ range of $(0.997-1.042) \mathrm{GeV}$ for $E_{\gamma}=4 \mathrm{GeV}$ and up to $-t$ of $0.2 \mathrm{GeV}^{2}$ an $M_{K \bar{K}}$ in the range $(1.01-1.03) \mathrm{GeV}$ at $E_{\gamma}=5.65 \mathrm{GeV}$, respectively.

\begin{tabular}{lcc}
\hline \hline Photon energy & $4.00 \mathrm{GeV}$ & $5.65 \mathrm{GeV}$ \\
\hline Sum of $P$ waves & $218.4 \pm 39.5$ & $120.5 \pm 9.4$ \\
Background & $300.0_{-10.7}^{+10.0}$ & $4.7_{-5.8}^{+4.2}$ \\
$P_{0}$ wave & $4.7_{-4.5}^{+5.7}$ & $14.0_{-4.8}^{+5.3}$ \\
$S$ wave & $4.3_{-3.6}^{+6.6}$ & $6.8_{-4.3}^{+6.6}$ \\
\hline \hline
\end{tabular}

\section{SUMMARY}

In summary, we performed a partial wave analysis of the reaction $\gamma p \rightarrow p K^{+} K^{-}$in the photon energy range 3.0-3.8 GeV and momentum transfer range $-t=0.6-1.3 \mathrm{GeV}^{2}$. Peripheral photoproduction of meson resonances is an important reaction to study their structure. On one side, photons have a pointlike coupling to quarks, which enhances production of compact states. On the other, pion exchange amplitudes in photoproduction on the nucleon can be used to determine rate of resonance production through final state interactions. Theoretical analysis of these processes are currently underway [45]. Moments of the dikaon angular distributions, defined as bilinear functions of the partial wave amplitudes, were fitted to the experimental data by means of an unbinned likelihood procedure. Different parametrization bases were used and detailed systematic checks were performed to ensure the reliability of the analysis procedure. We extracted moments $\left\langle Y_{L M}\right\rangle$ with $L \leq 4$ and $M \leq 2$ by using amplitudes with $L \leq 2$ (up to $P$ waves). The production amplitudes have been parametrized using a Regge-theory inspired model. The $P$ wave, dominated by the $\phi(1020)$ meson, was parametrized by Pomeron exchange, while the $f_{0}(980)$ meson in the $S$ wave was described by the exchange of the $\omega$ and $\rho$ vector mesons in the $t$ channel. This model also accounts for the final state interaction (FSI) of the emitted kaons. The moment $\left\langle Y_{00}\right\rangle$ is dominated by the $\phi(1020)$ meson contribution in the $P$ wave, while the moments $\left\langle Y_{10}\right\rangle$ and $\left\langle Y_{11}\right\rangle$ show contributions of the $S$ wave through interference with the $P$ wave. The cross sections of $S$ and $P$ waves in the mass range of the $\phi(1020)$ were computed. This is the first time the $t$-dependent cross section of the $S$-wave contribution to the elastic $K^{+} K^{-}$ photoproduction has been measured.

\section{ACKNOWLEDGMENTS}

We would like to acknowledge the outstanding efforts of the staff of the Accelerator and the Physics Divisions at Jefferson Lab that made this experiment possible. This work was supported in part by the Chilean Comisión 
Nacional de Investigación Científica y Tecnológica (CONICYT), the Italian Istituto Nazionale di Fisica Nucleare, the French Centre National de la Recherche Scientifique, the French Commissariat à l'Energie Atomique, the U.S. Department of Energy, the National Science Foundation, the Scottish Universities Physics Alliance (SUPA), the United Kingdom's Science and Technology Facilities Council, and the National Research Foundation of Korea. The Southeastern Universities Research Association (SURA) operates the Thomas Jefferson National Accelerator Facility for the United States Department of Energy under Contract No. DE-AC05-84ER40150. This material is based upon work supported by the U.S. Department of Energy, Office of Science, Office of Nuclear Physics under Contract No. DE-AC05-06OR23177.

\section{APPENDIX A: SYSTEMATIC STUDIES OF THE MOMENT EXTRACTION}

To check the results stability against the analysis procedure many systematic checks have been performed. The photon energy bin size has been split in two and the analysis repeated in the two bins independently finding no dependence in moment extractions. The $\Lambda(1520)$ peak in the $K^{-} p$ mass distribution cannot be reproduced with $\lambda_{\max }<4$ (see Fig. 18), but since this kinematical region is not a main focus of this study, the cut $M_{K^{-}} p 1.6 \mathrm{GeV}$ was applied throughout the analysis. Additional systematic checks showed that this cut did not affect the number of events in the $M_{K^{+} K^{-}}$region near the $\phi$ mass. The sensitivity to $\lambda_{\max }$ and effect of truncation to $\lambda_{\max }=4$ have also been studied comparing the $K^{+}$helicity angle distributions and $K^{+} p, K^{-} p$ invariant mass distributions obtained by the fit to the raw data. Accepted Monte Carlo events, with primary events generated from a flat phase-space distribution, took

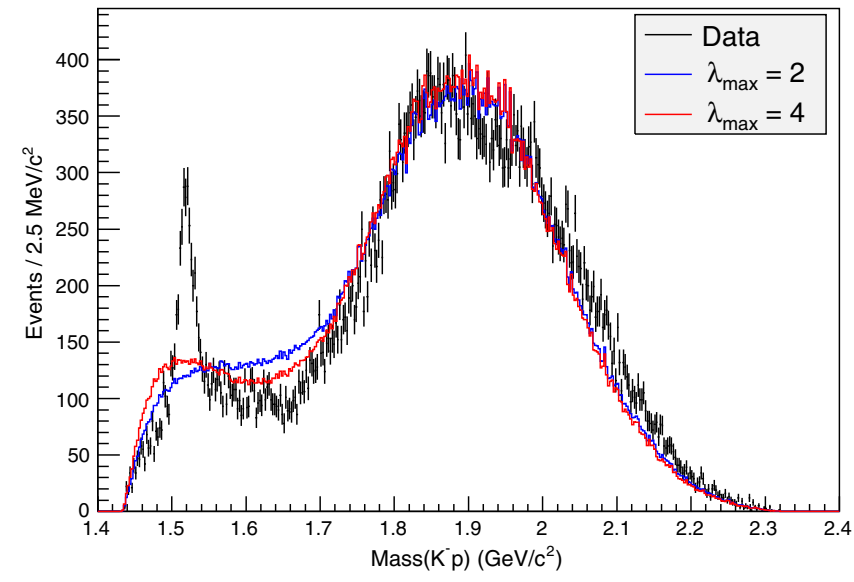

FIG. 18. Measured number of events as a function of the $p K^{-}$ invariant mass compared to the predicted distribution computed with fitted results from method 3 weighted by the experimental acceptance before cutting out the $\Lambda(1520)$. the same form as the data in this region due to the detector acceptance showing a good agreement between the two distributions.

\section{APPENDIX B: PARAMETRIZATION OF INDIVIDUAL $K^{+} K^{-}$AMPLITUDES}

We restricted our analysis to waves with $M \leq 1$ and partial waves up to $L=1$ waves.

\section{1. $P$ wave}

The $P$ waves were constructed based on the model of elastic $K^{+} K^{-}$photoproduction developed in [42]. The model assumes that the $\phi(1020)$ resonance is produced by a soft Pomeron exchange, which leads to an almost purely imaginary amplitude at small momentum transfers. The $K^{+} K^{-}$effective mass distribution is described by the relativistic Breit-Wigner formula

$$
\mathrm{BW}\left(M_{K^{+} K^{-}}\right)=\frac{1}{M_{\phi}^{2}-M_{K^{+} K^{-}}^{2}-i M_{\phi} \Gamma_{\phi}},
$$

with $M_{\phi}$ and $\Gamma_{\phi}$ being the $\phi$ meson mass and width. Expanding the $P$-wave amplitudes into partial waves,

$$
f_{\sigma, \lambda, \lambda^{\prime}}^{1}(s, t, W, \Omega)=\sum_{M} f_{\sigma, \lambda, \lambda^{\prime}}^{1 M}(s, t, W) Y_{1 M}(\Omega),
$$

and taking the high-energy limit, $s \gg t$ and $s \gg M_{K^{+} K^{-}}^{2}$, the amplitudes derived in [42] result in the following helicity partial waves:

$f_{+++}^{1,1}=f_{+--}^{1,1} \propto s \sqrt{M_{K^{+} K^{-}}^{2}-4 m_{K}^{2}} \mathrm{BW}\left(M_{K^{+} K^{-}}\right)$,

$f_{+++}^{1,0}=f_{+--}^{1,0} \propto s \sqrt{-t} \sqrt{M_{K^{+} K^{-}}^{2}-4 m_{K}^{2}} \mathrm{BW}\left(M_{K^{+} K^{-}}\right)$.

Before comparing with data we multiplied each of these amplitudes by a slowly varying function of $M_{K^{+} K^{-}}$,

$$
f\left(M_{K^{+} K^{-}}\right)=a+b w\left(M_{K^{+} K^{-}}\right)+c w^{2}\left(M_{K^{+} K^{-}}\right)
$$

with $w(z)$ conformally mapping the complex $M_{K^{+} K^{-}}^{2}$ plane cut at $M_{K K}^{2}=0$ and $M_{K K}^{2}=4 m_{K}^{2}$ onto a unit circle. Coefficients $a, b$ and $c$ are allowed to vary independently for each helicity amplitude.

\section{2. $S$ wave}

The $S$-wave component of the $K^{+} K^{-}$amplitude is parametrized by the double $t$-channel exchange of the $\rho$ and $\omega$ vector mesons as described in [44]. In the upper meson vertex, a simple meson exchange is used, allowing for an interaction of two produced mesons in the final state. 
The normal propagator $\left(t-m_{e}^{2}\right)^{-1}$, where $m_{e}$ is the mass of the exchanged vector meson, was used at the nucleon vertex. Both the $\pi^{+} \pi^{-}$and $K^{+} K^{-}$channels were included in the final state interactions. The $S$ wave in the mass region considered is dominated by the $f_{0}(980)$ and $a_{0}(980)$ resonances. Each partial-wave helicity $S$-wave amplitude was multiplied by the function $f\left(M_{K^{+} K^{-}}\right)$given in Eq. (B5), which contains three independent fit parameters.
[1] O. Bartholomy et al., Phys. Rev. Lett. 94, 012003 (2005).

[2] V. Crede et al., Phys. Rev. Lett. 94, 012004 (2005).

[3] I. Horn et al., Eur. Phys. J. A 38, 173 (2008).

[4] E. F. McNikoll et al., Phys. Rev. C 82, 035208 (2010).

[5] T. C. Jude et al., Phys. Lett. B 735, 112 (2014).

[6] M. Ostrick (MAMI Collaboration), J. Phys. Soc. Jpn. Conf. Proc. 10, 010004 (2016).

[7] T. Mibe et al., Phys. Rev. Lett. 95, 182001 (2005).

[8] K. Hicks et al., Phys. Rev. Lett. 102, 012501 (2009).

[9] K. Mizutani et al., Phys. Rev. C 96, 062201 (2017).

[10] R. Brandford et al., Phys. Rev. C 73, 035202 (2006).

[11] M. Williams et al., Phys. Rev. C 80, 045213 (2009).

[12] D. Ireland (CLAS Collaboration), Proc. Sci. INPC2016 (2017) 265.

[13] A. Austregesilo et al., Int. J. Mod. Phys. Conf. Ser. 46, 1860029 (2018).

[14] M. Battaglieri et al., JLab approved experiment E12-11-005 (2011); A. Celentano, Acta Phys. Pol. B Proc. Suppl. 6, 769 (2013).

[15] J. Ballam et al., Phys. Rev. D 7, 3150 (1973).

[16] M. Battaglieri, Prog. Part. Nucl. Phys. 67, 603 (2012).

[17] J. R. Pelaez, Phys. Rep. 658, 1 (2016).

[18] R. Kaminski, J. R. Pelaez, and F. J. Yndurain, Phys. Rev. D 74, 014001 (2006).

[19] I. Caprini, G. Colangelo, and H. Leutwyler, Phys. Rev. Lett. 96, 132001 (2006).

[20] R. Kaminski, J. R. Pelaez, and F. J. Yndurain, Phys. Rev. D 77, 054015 (2008).

[21] L. Y. Dai and M. R. Pennington, Phys. Rev. D 90, 036004 (2014).

[22] R. A. Briceno, J. J. Dudek, R. G. Edwards, and D. J. Wilson, Phys. Rev. D 97, 054513 (2018).

[23] D. Aston et al., Nucl. Phys. B172, 1 (1980).

[24] D. C. Fries, P. Heine, H. Hirschmann, A. Markou, E. Seitz, H.-J. Behrend, W. P. Hesse, W. A. McNeely, and T. Miyachi, Nucl. Phys. B143, 408 (1978).

[25] B. Dey et al. (CLAS Collaboration), Phys. Rev. C 89, 055208 (2014).
[26] H.-J. Behrend, J. Bodenkamp, W. P. Hesse, W. A. McNeely, T. Miyachi, D. C. Fries, P. Heine, H. Hirschmann, A. Markou, and E. Seitz, Nucl. Phys. B144, 22 (1978).

[27] D. P. Barber et al., Z. Phys. C 12, 1 (1982).

[28] R. Aaij et al. (CLHCb Collaboration), Phys. Rev. D 87, 072004 (2013).

[29] M. Battaglieri et al. (CLAS Collaboration), Phys. Rev. Lett. 102, 102001 (2009).

[30] M. Battaglieri et al. (CLAS Collaboration), Phys. Rev. D 80, 072005 (2009).

[31] B. A. Mecking et al., Nucl. Instrum. Methods Phys. Res., Sect. A 503, 513 (2003).

[32] D. I. Sober et al., Nucl. Instrum. Methods Phys. Res., Sect. A 440, 263 (2000).

[33] S. Stepanyan et al., Nucl. Instrum. Methods Phys. Res., Sect. A 572, 654 (2007).

[34] R. De Vita et al. (CLAS Collaboration), Phys. Rev. D 74, 032001 (2006).

[35] M. D. Mestayer et al., Nucl. Instrum. Methods Phys. Res., Sect. A 449, 81 (2000).

[36] E. S. Smith et al., Nucl. Instrum. Methods Phys. Res., Sect. A 432, 265 (1999).

[37] Y. G. Sharabian et al., Nucl. Instrum. Methods Phys. Res., Sect. A 556, 246 (2006).

[38] S. U. Chung, Phys. Rev. D 56, 7299 (1997).

[39] S. Lombardo, CLAS-Analysis Report No. 2017-007, https://misportal.jlab.org/ul/Physics/Hall-B/clas/viewFile .cfm/2017-007.pdf?documentId=775.

[40] JLab Experiment CLAS Database, Jefferson Lab, http:// clasweb.jlab.org/physicsdb/intro.html.

[41] The Durham HEP Databases, http://durpdg.dur.ac.uk/.

[42] L. Lesniak and A. P. Szczepaniak, Acta Phys. Pol. B 34, 3389 (2003).

[43] A. C. Irving and R. P. Worden, Phys. Rep. 34, 117 (1977).

[44] L. Bibrzycki, L. Lesniak, and A. P. Szczepaniak, Eur. Phys. J. C 34, 335 (2004).

[45] L. Bibrzycki and R. Kaminski, Phys. Rev. D 87, 114010 (2013). 